\title{
Codes and Designs Related to Lifted MRD Codes
}

\author{
Tuvi Etzion, Fellow, IEEE and Natalia Silberstein
}

\begin{abstract}
Lifted maximum rank distance (MRD) codes, which are constant dimension codes, are considered. It is shown that a lifted MRD code can be represented in such a way that it forms a block design known as a transversal design. A slightly different representation of this design makes it similar to a $q$-analog of a transversal design. The structure of these designs is used to obtain upper bounds on the sizes of constant dimension codes which contain a lifted MRD code. Codes which attain these bounds are constructed. These codes are the largest known codes for the given parameters. These transversal designs can be also used to derive a new family of linear codes in the Hamming space. Bounds on the minimum distance and the dimension of such codes are given.
\end{abstract}

Index Terms - constant dimension codes, Grassmannian space, lifted MRD codes, rank-metric codes, transversal designs.

\section{INTRODUCTION}

$\mathbf{L}$ ET $\mathbb{F}_{q}$ be the finite field of size $q$. For two $k \times \ell$ matrices $A$ and $B$ over $\mathbb{F}_{q}$ the rank distance is defined by

$$
d_{R}(A, B) \stackrel{\text { def }}{=} \operatorname{rank}(A-B) .
$$

A $[k \times \ell, \varrho, \delta]$ rank-metric code $\mathcal{C}$ is a linear code, whose codewords are $k \times \ell$ matrices over $\mathbb{F}_{q}$; they form a linear subspace with dimension $\varrho$ of $\mathbb{F}_{q}^{k \times \ell}$, and for each two distinct codewords $A$ and $B$ we have that $d_{R}(A, B) \geq \delta$. For a $[k \times \ell, \varrho, \delta]$ rank-metric code $\mathcal{C}$ it was proved in [10], [17], [35] that

$$
\varrho \leq \min \{k(\ell-\delta+1), \ell(k-\delta+1)\} .
$$

This bound, called Singleton bound for the rank metric, is attained for all feasible parameters. The codes which attain this bound are called maximum rank distance codes (or MRD codes in short).

Rank-metric codes have found application in public key cryptosystems [18], space-time coding [32], authentication codes [52], rank-minimization over finite fields [44], and distributed storage systems [41]. Recently, rank-metric codes also have found a new application in the construction of errorcorrecting codes for random network coding [42]. For this application, the $k \times \ell$ matrices are lifted into $k$-dimensional subspaces of $\mathbb{F}_{q}^{k+\ell}$ [42] as described below.

T. Etzion is with the Department of Computer Science, Technion - Israel Institute of Technology, Haifa 32000, Israel. (email: etzion@cs.technion.ac.il).

N. Silberstein is with the Department of Electrical and Computer Engineering, University of Texas at Austin, Austin, TX 78712-1684, USA (email:natalys@austin.utexas.edu). This work was done when she was with the Department of Computer Science, Technion - Israel Institute of Technology, Haifa 32000, Israel. This work is part of her Ph.D. thesis performed at the Technion.

The material in this paper was presented in part in the 2011 IEEE International Symposium on Information Theory, Saint Petersburg, Russia, August 2011.

This work was supported in part by the Israel Science Foundation (ISF), Jerusalem, Israel, under Grant 230/08.
Let $A$ be a $k \times \ell$ matrix over $\mathbb{F}_{q}$ and let $I_{k}$ be a $k \times k$ identity matrix. The matrix $\left[I_{k} A\right]$ can be viewed as a generator matrix of a $k$-dimensional subspace of $\mathbb{F}_{q}^{k+\ell}$, and it is called the lifting of $A$ [42].

Example 1: Let $A$ and $\left[\begin{array}{ll}I_{3} & A\end{array}\right]$ be the following matrices over $\mathbb{F}_{2}$

$$
A=\left(\begin{array}{lll}
1 & 1 & 0 \\
0 & 1 & 1 \\
0 & 0 & 1
\end{array}\right),\left[\begin{array}{ll}
I_{3} & A
\end{array}\right]=\left(\begin{array}{llllll}
1 & 0 & 0 & 1 & 1 & 0 \\
0 & 1 & 0 & 0 & 1 & 1 \\
0 & 0 & 1 & 0 & 0 & 1
\end{array}\right)
$$

then the subspace obtained by the lifting of $A$ is given by the following 8 vectors:

$$
\begin{aligned}
& \text { (100110), (010011), (001001), (110101), } \\
& \text { (101111), (011010), (111100), (000000). }
\end{aligned}
$$

Given a nonnegative integer $k \leq n$, the set of all $k$-dimensional subspaces of $\mathbb{F}_{q}^{n}$ forms the Grassmannian space (Grassmannian in short) over $\mathbb{F}_{q}$, which is denoted by $\mathcal{G}_{q}(n, k)$. It is well known that $\left|\mathcal{G}_{q}(n, k)\right|=\left[\begin{array}{l}n \\ k\end{array}\right]_{q}=$ $\prod_{i=0}^{k-1} \frac{q^{n-i}-1}{q^{k-i}-1}$, where $\left[\begin{array}{l}n \\ k\end{array}\right]_{q}$ is the $q$-ary Gaussian coefficient.

A subset $\mathbb{C}$ of $\mathcal{G}_{q}(n, k)$ is called an $\left(n, M, d_{S}, k\right)_{q}$ constant dimension code if it has size $M$ and minimum subspace distance $d_{S}$, where the distance function in $\mathcal{G}_{q}(n, k)$ is defined by

$$
d_{S}(X, Y) \stackrel{\text { def }}{=} \operatorname{dim} X+\operatorname{dim} Y-2 \operatorname{dim}(X \cap Y),
$$

for any two subspaces $X$ and $Y$ in $\mathcal{G}_{q}(n, k) . \mathcal{A}_{q}(n, d, k)$ will denote the maximum size of an $(n, M, d, k)_{q}$ code.

Codes in the Grassmannian gained recently lot of interest due to the work by Koetter and Kschischang [26], where they presented an application of such codes for error-correction in random network coding. When the codewords of a rank-metric code $\mathcal{C}$ are lifted to $k$-dimensional subspaces, the result is a constant dimension code $\mathbb{C}$. If $\mathcal{C}$ is an MRD code then $\mathbb{C}$ is called a lifted MRD code [42]. This code will be denoted by $\mathbb{C}^{\mathrm{MRD}}$.

Theorem 1: [42] Let $k, n$ be positive integers such that $k \leq n-k$. If $\mathcal{C}$ is a $[k \times(n-k),(n-k)(k-\delta+1), \delta]$ MRD code then $\mathbb{C}^{\mathrm{MRD}}$ is an $\left(n, q^{(n-k)(k-\delta+1)}, 2 \delta, k\right)_{q}$ code.

In view of Theorem 1 we will assume throughout the paper that that $1<k \leq n-k$. $\mathbb{C}^{\mathrm{MRD}}$ which is an $\left(n, q^{(n-k)(k-\delta+1)}, 2 \delta, k\right)_{q}$ code will be also called an $(n, k, \delta)_{q}$ $\mathbb{C}^{\mathrm{MRD}}$. If no parameters for $\mathbb{C}^{\mathrm{MRD}}$ will be given we will assume it is an $(n, k, \delta)_{q} \mathbb{C}^{\mathrm{MRD}}$.

Most of the constructions for large constant dimension codes known in the literature produce codes which contain $\mathbb{C}^{\mathrm{MRD}}$ [13], [20], [33], [39], [42], [43], [48]. The only constructions which generate codes that do not contain $\mathbb{C}^{\mathrm{MRD}}$ are given in [15], [27], [49]. These constructions are either of so called 
orbit codes or specific constructions for small parameters. Moreover, only $(n, M, d, 3)_{2}$ orbit codes (specifically cyclic codes) with $8 \leq n \leq 12$, and $(6,77,4,3)_{2}$ and $(7,304,4,3)_{2}$ codes are the largest codes for their specific parameters which do not contain $\mathbb{C}^{\mathrm{MRD}}$ [27]. This motivates the question, what is the largest constant dimension code which contain $\mathbb{C}^{\mathrm{MRD}}$ ?

The well-known concept of $q$-analogs replaces subsets by subspaces of a vector space over a finite field and their orders by the dimensions of the subspaces. In particular, the $q$-analog of a constant weight code in the Johnson space is a constant dimension code in the Grassmannian space. Related to constant dimension codes are $q$-analogs of block designs. $q$-analogs of designs were studied in [1], [7], [15], [16], [37], [47]. For example, in [1] it was shown that Steiner structures (the $q$-analog of Steiner system), if exist, yield optimal codes in the Grassmannian. Another connection is the constructions of constant dimension codes from spreads which are given in [15] and [33].

In this paper we consider several topics related to lifted MRD codes. First, we discuss properties of these codes related to block designs. We prove that the codewords of $\mathbb{C}^{\mathrm{MRD}}$ form a design called a transversal design, a structure which is known to be equivalent to the well known orthogonal array. We also prove that the same codewords form a subspace transversal design, which is akin to the transversal design, but not its $q$-analog.

The structure of $\mathbb{C}^{\mathrm{MRD}}$ as a transversal design leads to the other results given in this paper. We derive for new lower bounds on $\mathcal{A}_{q}(n, d, k)$ and upper bounds on the sizes of errorcorrecting constant dimension codes which contain $\mathbb{C}^{\mathrm{MRD}}$. In particular, we prove that if an $(n, M, 2(k-1), k)_{q}$ code $\mathbb{C}$, $k \geq 3$, contains an $(n, k, k-1)_{q} \mathbb{C}^{\mathrm{MRD}}$ code then

$$
M \leq q^{2(n-k)}+\mathcal{A}_{q}(n-k, 2(k-2), k-1) .
$$

We present a construction for codes which either attain this bound or almost attain it for $k=3$. These codes are the largest known $(n, M, 4,3)_{q}$ codes for $n \geq 13$.

We prove that if an $(n, M, 2 k, 2 k)_{q}$ code $\mathbb{C}$ contains an $(n, 2 k, k)_{q} \mathbb{C}^{\mathrm{MRD}}$ code then

$M \leq q^{(n-2 k)(k+1)}+\left[\begin{array}{c}n-2 k \\ k\end{array}\right]_{q} \frac{q^{n}-q^{n-2 k}}{q^{2 k}-q^{k}}+\mathcal{A}_{q}(n-2 k, 2 k, 2 k)$.

We present a construction for codes which attain this bound when $2 k=4, n=8$, and for all $q$. These codes are the largest known for the related parameters.

The incidence matrix of the transversal design derived from $\mathbb{C}^{\mathrm{MRD}}$ can be viewed as a parity-check matrix of a linear code in the Hamming space. This way to construct a linear code from a design is well-known [2], [12], [23], [25], [28], [29], [30], [50], [51], [55]. We find the properties of these codes, in particular, we present the bounds on their minimum distance and dimension.

The rest of this paper is organized as follows. In Section we present properties of lifted MRD codes. Then we prove that these codes form transversal designs in sets and subspaces. In Section III we discuss some known upper bounds on $\mathcal{A}_{q}(n, d, k)$ and present two new upper bounds on the sizes of constant dimension codes which contain $\mathbb{C}^{\mathrm{MRD}}$. In Sections IV and $\mathrm{V}$ we provide constructions of two families of codes that attain the upper bounds of Section III. In Section VI we consider properties of linear codes whose parity-check matrices are derived from $\mathbb{C}^{\mathrm{MRD}}$. Conclusions and problems for future research are given in Section VII

\section{LIFTED MRD CODES AND TRANSVERSAL DESIGNS}

In this section we prove that a lifted MRD code yield a combinatorial structure known as a transversal design. Moreover, the codewords of these codes form the blocks of a new type of transversal design, called a subspace transversal design. Based on these designs, we will present some novel results in the following sections. We first examine some combinatorial properties of lifted MRD codes. Based on these properties we will construct the transversal designs.

\section{A. Properties of lifted MRD codes}

Let $\mathcal{L}^{(n, k)}$ be the set of $q^{n}-q^{n-k}$ vectors of length $n$ over $\mathbb{F}_{q}$ in which not all the first $k$ entries are zeroes. The following lemma is a simple observation.

Lemma 2: All the nonzero vectors which are contained in codewords of an $(n, k, \delta)_{q} \mathbb{C}^{\mathrm{MRD}}$ belong to $\mathcal{L}^{(n, k)}$.

For a set $\mathcal{S} \subseteq \mathbb{F}_{q}^{n}$, let $\langle\mathcal{S}\rangle$ denotes the subspace of $\mathbb{F}_{q}^{n}$ spanned by the elements of $\mathcal{S}$. If $\mathcal{S}=\{v\}$ is of size one then we denote $\langle\mathcal{S}\rangle$ by $\langle v\rangle$. For $v_{1} \in \mathbb{F}_{q}^{n_{1}}$ and $v_{2} \in \mathbb{F}_{q}^{n_{2}}$ we denote by $v_{1} \| v_{2} \in \mathbb{F}_{q}^{n_{1}+n_{2}}$ the concatenation of $v_{1}$ and $v_{2}$. Let $\mathbb{V}^{n}=\left\{\langle v\rangle: v \in \mathcal{L}^{(n, k)}\right\}$ be the set of all $\frac{q^{n}-q^{n-k}}{q-1}$ one-dimensional subspaces of $\mathbb{F}_{q}^{n}$ whose nonzero vectors are contained in $\mathcal{L}^{(n, k)}$. We identify each subspace $A \in \mathcal{G}_{q}(\ell, 1)$, for any given $\ell$, with the vector $v_{A} \in A$ (of length $\ell$ ) in which the first nonzero entry is a one.

For each $A \in \mathcal{G}_{q}(k, 1)$ we define

$$
\mathbb{V}_{A}^{(n, k)} \stackrel{\text { def }}{=}\left\{X \mid X=\langle v\rangle, v=v_{A} \| z, z \in \mathbb{F}_{q}^{n-k}\right\},
$$

in other words, $\mathbb{V}_{A}^{(n, k)}$ consists of all one-dimensional subspaces whose restriction to the first $k$ coordinates is precisely $A .\left\{\mathbb{V}_{A}^{(n, k)}: A \in \mathcal{G}_{q}(k, 1)\right\}$ contains $\frac{q^{k}-1}{q-1}$ sets, each one of the size $q^{n-k}$. These sets partition the set $\mathbb{V}^{n}$, i.e.,

$$
\mathbb{V}_{A}^{(n, k)} \cap \mathbb{V}_{B}^{(n, k)}=\varnothing, A, B \in \mathcal{G}_{q}(k, 1), A \neq B
$$

and

$$
\mathbb{V}^{n}=\bigcup_{A \in \mathcal{G}_{q}(k, 1)} \mathbb{V}_{A}^{(n, k)}
$$

We say that a vector $v \in \mathbb{F}_{q}^{n}$ is in $\mathbb{V}_{A}^{(n, k)}$ if $v \in X$ for $X \in \mathbb{V}_{A}^{(n, k)}$. Clearly, $\left\langle\left\{v_{A}\left\|z^{\prime}, v_{A}\right\| z^{\prime \prime}\right\}\right\rangle$, for $A \in \mathcal{G}_{q}(k, 1)$ and $z^{\prime} \neq z^{\prime \prime}$, contains a vector with $k$ leading zeroes. Such a vector does not belong to $\mathcal{L}^{(n, k)}$ and hence, by Lemma 2 we have

Lemma 3: For each $A \in \mathcal{G}_{q}(k, 1)$, a codeword of $\mathbb{C}^{\mathrm{MRD}}$ contains at most one element from $\mathbb{V}_{A}^{(n, k)}$.

Note that each $k$-dimensional subspace of $\mathbb{F}_{q}^{n}$ contains $\left[\begin{array}{l}k \\ 1\end{array}\right]_{q}=\frac{q^{k}-1}{q-1}$ one-dimensional subspaces. Therefore, by Lemma 2, each codeword of $\mathbb{C}^{\mathrm{MRD}}$ contains $\frac{q^{k}-1}{q-1}$ elements 
of $\mathbb{V}^{n}$. Hence, by Lemma 3 and since $\left|\mathcal{G}_{q}(k, 1)\right|=\frac{q^{k}-1}{q-1}$ we have

Corollary 1: For each $A \in \mathcal{G}_{q}(k, 1)$, a codeword of $\mathbb{C}^{\mathrm{MRD}}$ contains exactly one element from $\mathbb{V}_{A}^{(n, k)}$.

Lemma 4: Each $(k-\delta+1)$-dimensional subspace $Y$ of $\mathbb{F}_{q}^{n}$, whose nonzero vectors are contained in $\mathcal{L}^{(n, k)}$, is contained in exactly one codeword of an $(n, k, \delta)_{q} \mathbb{C}^{\mathrm{MRD}}$.

Proof: Let

$$
\mathbb{S} \stackrel{\text { def }}{=}\left\{Y \in \mathcal{G}_{q}(n, k-\delta+1):\left|Y \cap \mathcal{L}^{(n, k)}\right|=q^{k-\delta+1}-1\right\},
$$

i.e. $\mathbb{S}$ consists of all $(k-\delta+1)$-dimensional subspaces of $\mathbb{F}_{q}^{n}$ in which all the nonzero vectors are contained in $\mathcal{L}^{(n, k)}$.

Since the minimum distance of $\mathbb{C}^{\mathrm{MRD}}$ is $2 \delta$ and its codewords are $k$-dimensional subspaces, it follows that the intersection of any two codewords is at most of dimension $k-\delta$. Hence, each $(k-\delta+1)$-dimensional subspace of $\mathbb{F}_{q}^{n}$ is contained in at most one codeword. The size of $\mathbb{C}^{\mathrm{MRD}}$ is $q^{(n-k)(k-\delta+1)}$, and the number of $(k-\delta+1)$-dimensional subspaces in a codeword is exactly $\left[\begin{array}{c}k \\ k-\delta+1\end{array}\right]_{q}$. By Lemma 2 each $(k-\delta+1)$-dimensional subspace, of a codeword, is contained in $\mathbb{S}$. Hence, the codewords of $\mathbb{C}^{\mathrm{MRD}}$ contain exactly $\left[\begin{array}{c}k \\ k-\delta+1\end{array}\right]_{q} q^{(n-k)(k-\delta+1)}$ distinct $(k-\delta+1)$-dimensional subspaces of $\mathbb{S}$.

To complete the proof we only have to show that $\mathbb{S}$ does not contain more $(k-\delta+1)$-dimensional subspaces. Hence, we will compute the size of $\mathbb{S}$. Each element of $\mathbb{S}$ intersects with each $\mathbb{V}_{A}^{(n, k)}, A \in \mathcal{G}_{q}(k, 1)$ in at most one one-dimensional subspace (since it contains vectors only from $\mathcal{L}^{(n, k)}$ ). There are $\left[\begin{array}{c}k \\ k-\delta+1\end{array}\right]_{q}$ ways to choose an arbitrary $(k-\delta+1)$ dimensional subspace of $\mathbb{F}_{q}^{k}$. For each such subspace we choose a basis $\left\{x_{1}, x_{2}, \ldots, x_{k-\delta+1}\right\}$, where each $x_{i}$ belongs to a different set $\mathbb{V}_{A}^{(n, k)}, A \in \mathcal{G}_{q}(k, 1)$ (clearly, by previous definition, in each such basis vector the first nonzero entry is a one). A basis for a $(k-\delta+1)$-dimensional subspace of $\mathbb{S}$ will be generated by concatenation of $x_{i}$ with a vector $z \in \mathbb{F}_{q}^{n-k}$ for each $i, 1 \leq i \leq k-\delta+1$. Therefore, there are $q^{(n-k)(k-\delta+1)}$ ways to choose a basis for an element of $\mathbb{S}$. Hence, $|\mathbb{S}|=\left[\begin{array}{c}k \\ k-\delta+1\end{array}\right]_{q} q^{(n-k)(k-\delta+1)}$.

Thus, the lemma follows.

Corollary 2: For each $i, 0 \leq i \leq k-\delta-1$, each $(k-\delta-i)$ dimensional subspace of $\mathbb{F}_{q}^{n}$, whose nonzero vectors are contained in $\mathcal{L}^{(n, k)}$, is contained in exactly $q^{(n-k)(i+1)}$ codewords of $\mathbb{C}^{\mathrm{MRD}}$.

Proof: The size of $\mathbb{C}^{\mathrm{MRD}}$ is $q^{(n-k)(k-\delta+1)}$. The number of $(k-\delta-i)$-dimensional subspaces in a codeword is exactly $\left[\begin{array}{c}k \\ k-\delta-i\end{array}\right]_{q}$. Hence, the total number of $(k-\delta-i)$-dimensional subspaces in $\mathbb{C}^{\mathrm{MRD}}$ is $\left[\begin{array}{c}k \\ k-\delta-i\end{array}\right]_{q} q^{(n-k)(k-\delta+1)}$ (clearly, each such $(k-\delta-i)$ dimensional subspace is counted more than once in this computation). Similarly to the proof of Lemma 4 we can prove that the total number of $(k-\delta-i)$-dimensional subspaces which contain nonzero vectors only from $\mathcal{L}^{(n, k)}$ is $\left[\begin{array}{c}k \\ k-\delta-i\end{array}\right]_{q} q^{(n-k)(k-\delta-i)}$. By simple symmetry, each two such subspaces are contained in the same number of codewords of $\mathbb{C}^{\mathrm{MRD}}$. Thus, each $(k-\delta-i)$-dimensional subspace of $\mathbb{F}_{q}^{n}$, whose nonzero vectors are contained in $\mathcal{L}^{(n, k)}$, is contained in exactly

$$
\frac{\left[\begin{array}{c}
k \\
k-\delta-i
\end{array}\right]_{q} q^{(n-k)(k-\delta+1)}}{\left[\begin{array}{c}
k \\
k-\delta-i
\end{array}\right]_{q} q^{(n-k)(k-\delta-i)}}=q^{(n-k)(i+1)}
$$

codewords of $\mathbb{C}^{\mathrm{MRD}}$.

Corollary 3: Each one-dimensional subspace $X \in \mathbb{V}^{n}$ is contained in exactly $q^{(n-k)(k-\delta)}$ codewords of $\mathbb{C}^{\mathrm{MRD}}$.

By applying Corollary 2 with $k-\delta-i=2$ we also infer the following result.

Corollary 4: Any two elements $X_{1}, X_{2} \in \mathbb{V}^{n}$, such that $X_{1} \in \mathbb{V}_{A}^{(n, k)}$ and $X_{2} \in \mathbb{V}_{B}^{(n, k)}, A \neq B$, are contained in exactly $q^{(n-k)(k-\delta-1)}$ codewords of $\mathbb{C}^{\mathrm{MRD}}$.

For the following lemma we need a generalization of the definition of a rank-metric code to a nonlinear rank-metric code, which is a subset of $\mathbb{F}_{q}^{k \times \ell}$ with minimum distance $\delta$ and size $q^{\varrho}$. If $\varrho=\min \{k(\ell-\delta+1), \ell(k-\delta+1)\}$, then such a code will be also called an MRD code.

Lemma 5: $\mathbb{C}^{\mathrm{MRD}}$ can be partitioned into $q^{(n-k)(k-\delta)}$ sets, called parallel classes, each one of size $q^{n-k}$, such that in each parallel class each element of $\mathbb{V}^{n}$ is contained in exactly one codeword.

Proof: First we prove that a lifted MRD code contains a lifted MRD subcode with disjoint codewords (subspaces). Let $G$ be the generator matrix of a $[k \times(n-k),(n-k)(k-\delta+1), \delta]$ MRD code $\mathcal{C}$ [17], $n-k \geq k$. Then $G$ has the following form

$$
G=\left(\begin{array}{cccc}
g_{1} & g_{2} & \ldots & g_{k} \\
g_{1}^{q} & g_{2}^{q} & \ldots & g_{k}^{q} \\
\vdots & \vdots & \ldots & \vdots \\
g_{1}^{q^{k-\delta}} & g_{2}^{q^{k-\delta}} & \ldots & g_{k}^{q^{k-\delta}}
\end{array}\right),
$$

where $g_{i} \in \mathbb{F}_{q^{n-k}}$ are linearly independent over $\mathbb{F}_{q}$. If the last $k-\delta$ rows are removed from $G$, the result is an MRD subcode of $\mathcal{C}$ with the minimum distance $k$. In other words, an $[k \times(n-k), n-k, k]$ MRD subcode $\tilde{\mathcal{C}}$ of $\mathcal{C}$ is obtained. The corresponding lifted code is an $\left(n, q^{n-k}, 2 k, k\right)_{q}$ lifted MRD subcode of $\mathbb{C}^{\mathrm{MRD}}$.

Let $\tilde{\mathcal{C}}_{1}=\tilde{\mathcal{C}}, \quad \tilde{\mathcal{C}}_{2}, \ldots, \tilde{\mathcal{C}}_{q^{(n-k)(k-\delta)}}$ be the $q^{(n-k)(k-\delta)}$ cosets of $\tilde{\mathcal{C}}$ in $\mathcal{C}$. All these $q^{(n-k)(k-\delta)}$ cosets are nonlinear rank-metric codes with the same parameters as the $[k \times(n-k), n-k, k]$ MRD code. Therefore, their lifted codes form a partition of an $(n, k, \delta)_{q} \mathbb{C}^{\mathrm{MRD}}$ into $q^{(n-k)(k-\delta)}$ parallel classes each one of size $q^{n-k}$, such that each element of $\mathbb{V}^{n}$ is contained in exactly one codeword of each parallel class.

\section{B. Transversal designs from lifted MRD codes}

A transversal design of groupsize $m$, blocksize $k$, strength $t$ and index $\lambda$, denoted by $\operatorname{TD}_{\lambda}(t, k, m)$ is a triple $(V, \mathcal{G}, \mathcal{B})$, where

1) $V$ is a set of $k m$ elements (called points);

2) $\mathcal{G}$ is a partition of $V$ into $k$ classes (called groups), each one of size $m$;

$3) \mathcal{B}$ is a collection of $k$-subsets of $V$ (called blocks); 
4) each block meets each group in exactly one point;

5) each $t$-subset of points that meets each group in at most one point is contained in exactly $\lambda$ blocks.

When $t=2$, the strength is usually not mentioned, and the design is denoted by $\mathrm{TD}_{\lambda}(k, m) . \mathrm{A}_{\lambda}(t, k, m)$ is resolvable if the set $\mathcal{B}$ can be partitioned into sets $\mathcal{B}_{1}, \ldots, \mathcal{B}_{s}$, where each element of $V$ is contained in exactly one block of each $\mathcal{B}_{i}$. The sets $\mathcal{B}_{1}, \ldots, \mathcal{B}_{s}$ are called parallel classes.

Example 2: Let $V=\{1,2, \ldots, 12\} ; \mathcal{G}=\left\{G_{1}, G_{2}, G_{3}\right\}$, where $G_{1}=\{1,2,3,4\}, G_{2}=\{5,6,7,8\}$, and $G_{3}=$ $\{9,10,11,12\} ; \mathcal{B}=\left\{B_{1}, B_{2}, \ldots, B_{16}\right\}$, where $B_{1}=$ $\{1,5,9\}, B_{2}=\{2,8,11\}, B_{3}=\{3,6,12\}, B_{4}=\{4,7,10\}$, $B_{5}=\{1,6,10\}, B_{6}=\{2,7,12\}, B_{7}=\{3,5,11\}$, $B_{8}=\{4,8,9\}, B_{9}=\{1,7,11\}, B_{10}=\{2,6,9\}, B_{11}=$ $\{3,8,10\}, B_{12}=\{4,5,12\}, B_{13}=\{1,8,12\}, B_{14}=$ $\{2,5,10\}, B_{15}=\{3,7,9\}$, and $B_{16}=\{4,6,11\}$. These blocks form a resolvable $T D_{1}(3,4)$ with four parallel classes $\mathcal{B}_{1}=\left\{B_{1}, B_{2}, B_{3}, B_{4}\right\}, \mathcal{B}_{2}=\left\{B_{5}, B_{6}, B_{7}, B_{8}\right\}, \mathcal{B}_{3}=$ $\left\{B_{9}, B_{10}, B_{11}, B_{12}\right\}$, and $\mathcal{B}_{4}=\left\{B_{13}, B_{14}, B_{15}, B_{16}\right\}$.

Theorem 6: The codewords of an $(n, k, \delta)_{q} \mathbb{C}^{\mathrm{MRD}}$ form the blocks of a resolvable transversal design $\operatorname{TD}_{\lambda}\left(\frac{q^{k}-1}{q-1}, q^{n-k}\right)$, $\lambda=q^{(n-k)(k-\delta-1)}$, with $q^{(n-k)(k-\delta)}$ parallel classes, each one of size $q^{n-k}$

Proof: Let $\mathbb{V}^{n}$ be the set of $\frac{q^{n}-q^{n-k}}{q-1}$ points for the design. Each set $\mathbb{V}_{A}^{(n, k)}, A \in \mathcal{G}_{q}(k, 1)$, is defined to be a group, i.e., there are $\frac{q^{k}-1}{q-1}$ groups, each one of size $q^{n-k}$. The $k$-dimensional subspaces (codewords) of $\mathbb{C}^{\mathrm{MRD}}$ are the blocks of the design. By Corollary 1 each block meets each group in exactly one point. By Corollary 4 each 2-subset which meets each group in at most one point is contained in exactly $q^{(n-k)(k-\delta-1)}$ blocks. Finally, by Lemma5 the design is resolvable with $q^{(n-k)(k-\delta)}$ parallel classes, each one of size $q^{n-k}$.

An $N \times k$ array $\mathcal{A}$ with entries from a set of $s$ elements is an orthogonal array with $s$ levels, strength $t$ and index $\lambda$, denoted by $\mathrm{OA}_{\lambda}(N, k, s, t)$, if every $N \times t$ subarray of $\mathcal{A}$ contains each $t$-tuple exactly $\lambda$ times as a row. It is known [21] that a $\operatorname{TD}_{\lambda}(k, m)$ is equivalent to an orthogonal array $\mathrm{OA}_{\lambda}\left(\lambda \cdot m^{2}, k, m, 2\right)$.

A $[k \times(n-k),(n-k)(k-\delta+1), \delta]$ MRD code $\mathcal{C}$ is a maximum distance separable (MDS) code if it is viewed as a code of length $k$ over $\mathbb{F}_{q^{n-k}}$ [17]. Thus its codewords form an orthogonal array $\mathrm{OA}_{\lambda}\left(q^{(n-k)(k-\delta+1)}, k, q^{n-k}, k-\right.$ $\delta+1)$ with $\lambda=1$, which is also an orthogonal array $\mathrm{OA}_{\lambda}\left(q^{(n-k)(k-\delta+1)}, k, q^{n-k}, 2\right)$ with $\lambda=q^{(n-k)(k-\delta-1)}$ (see [21] for the connection between MDS codes and orthogonal arrays).

By the equivalence of transversal designs and orthogonal arrays, and by Theorem 6 an $(n, k, \delta)_{q}$ code $\mathbb{C}^{\mathrm{MRD}}$ induces an $\mathrm{OA}_{\lambda}\left(q^{(n-k)(k-\delta+1)}, \frac{q^{k}-1}{q-1}, q^{n-k}, 2\right)$ with $\lambda=q^{(n-k)(k-\delta-1)}$. These parameters are different from the ones obtained by viewing an MRD code as an MDS code.

Now we define a new type of transversal designs in terms of subspaces, which will be called a subspace transversal design. We will show that such a design is induced by the codewords of a lifted MRD code. Moreover, we will show that this design is useful to obtain upper bounds on the codes that contain the lifted MRD codes, and in a construction of large constant dimension codes.

Let $\mathbb{V}_{0}^{(n, k)}$ be a set of one-dimensional subspaces in $\mathcal{G}_{q}(n, 1)$, that contains only vectors starting with $k$ zeroes. Note that $\mathbb{V}_{0}^{(n, k)}$ is isomorphic to $\mathcal{G}_{q}(n-k, 1)$.

A subspace transversal design of groupsize $q^{m}, m=n-k$, block dimension $k$, and strength $t$, denoted by $\operatorname{STD}_{q}(t, k, m)$, is a triple $\left(\mathbb{V}^{n}, \mathbb{G}, \mathbb{B}\right)$, where

1) $\mathbb{V}^{n}$ is the subset of all elements of $\mathcal{G}_{q}(n, 1) \backslash \mathbb{V}_{0}^{(n, k)}$, $\left|\mathbb{V}^{n}\right|=\frac{\left(q^{k}-1\right)}{q-1} q^{m}$ (the points);

2) $\mathbb{G}$ is a partition of $\mathbb{V}^{n}$ into $\frac{q^{k}-1}{q-1}$ classes of size $q^{m}$ (the groups);

3) $\mathbb{B}$ is a collection of $k$-dimensional subspaces which contain only points from $\mathbb{V}^{n}$ (the blocks);

4) each block meets each group in exactly one point;

5) each $t$-dimensional subspace (with points from $\mathbb{V}^{n}$ ) which meets each group in at most one point is contained in exactly one block.

An $\operatorname{STD}_{q}(t, k, m)$ is resolvable if the set $\mathcal{B}$ can be partitioned into sets $\mathcal{B}_{1}, \ldots, \mathcal{B}_{s}$, where each one-dimensional subspace of $V$ is contained in exactly one block of each $\mathcal{B}_{i}$. The sets $\mathcal{B}_{1}, \ldots, \mathcal{B}_{s}$ are called parallel classes.

As a direct consequence form Lemma 4 and Theorem 6 we infer the following theorem.

Theorem 7: The codewords of an $(n, k, \delta)_{q} \mathbb{C}^{\mathrm{MRD}}$ form the blocks of a resolvable $\operatorname{STD}_{q}(k-\delta+1, k, n-k)$, with the set of points $\mathbb{V}^{n}$ and the set of groups $\mathbb{V}_{A}^{(n, k)}, A \in \mathcal{G}_{q}(k, 1)$, defined previously in this section.

Remark 1: There is no known nontrivial $q$-analog of a block design with $\lambda=1$ and $t>1$. An $\operatorname{STD}_{q}(t, k, m)$ is very close to such a design.

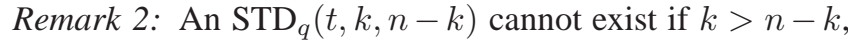
unless $t=k$. This is not difficult to prove and we leave it as an exercise for the interested reader. Recall, that the case $k>n-k$ was not considered in this section (see Theorem 1 ).

\section{UPPER BOUNDS ON THE SIZE OF CODES IN $\mathcal{G}_{q}(n, k)$}

In this section we consider upper bounds on the size of constant dimension codes. First, in Subsection ஹI-A we consider the Johnson type upper bound presented in [14], [15], [52], [53]. We estimate the size of known constant dimension codes relatively to this bound. The estimations provide better results than the ones known before, e.g. [26]. In Subsection III-B we provide new upper bounds on codes which contain lifted MRD codes. This type of upper bounds was not considered before, even so, as said before, usually the largest known codes contain the lifted MRD codes.

\section{A. Some known upper bounds}

Upper bounds on the sizes of constant dimension codes were obtained in several papers, e.g. [26], [42]. The following upper bound was established in [52] in the context of linear authentication codes and in [14], [15], [53] based on anticodes in the Grassmannian and as generalization of the well known Johnson bound for constant weight codes. 
Theorem 8:

$$
\mathcal{A}_{q}(n, 2 \delta, k) \leq \frac{\left[\begin{array}{c}
n \\
k-\delta+1
\end{array}\right]_{q}}{\left[\begin{array}{c}
k \\
k-\delta+1
\end{array}\right]_{q}}
$$

It was proved recently [6] that for fixed $q, k$, and $\delta$, the ratio between the upper bound of Theorem 8 and $\mathcal{A}_{q}(n, 2 \delta, k)$ equals to 1 as $n \rightarrow \infty$. But, the method used in [6] is based on probabilistic arguments and an explicit construction of the related code is not known. We will estimate the value of this upper bound.

$$
\begin{gathered}
\frac{\left[\begin{array}{c}
n \\
k-\delta+1
\end{array}\right]_{q}}{\left[\begin{array}{c}
k \\
k-\delta+1
\end{array}\right]_{q}}=\frac{\left(q^{n}-1\right)\left(q^{n-1}-1\right) \ldots\left(q^{n-k+\delta}-1\right)}{\left(q^{k}-1\right)\left(q^{k-1}-1\right) \ldots\left(q^{\delta}-1\right)} \\
=q^{(n-k)(k-\delta+1)} \frac{\left(1-q^{-n}\right)\left(1-q^{-n+1}\right) \ldots\left(1-q^{-n+k-\delta}\right)}{\left(1-q^{-k}\right)\left(1-q^{-k+1}\right) \ldots\left(1-q^{-\delta}\right)} \\
<\frac{q^{(n-k)(k-\delta+1)}}{\prod_{j=\delta}^{\infty}\left(1-q^{-j}\right)} .
\end{gathered}
$$

We define $Q_{\delta}(q)=\prod_{j=\delta}^{\infty}\left(1-q^{-j}\right), \delta \geq 1$. Similar analysis for $Q_{1}(q)$ was considered in [26] and $Q_{2}(q)$ was considered also in [19]. Since $(n, k, \delta)_{q} \mathbb{C}^{\mathrm{MRD}}$ has $q^{(n-k)(k-\delta+1)}$ codewords we have that

Lemma 9: The ratio between the size of an $(n, k, \delta)_{q} \mathbb{C}^{\mathrm{MRD}}$ and the upper bound on $\mathcal{A}_{q}(n, 2 \delta, k)$ given in (2) satisfies

$$
\frac{\left|\mathbb{C}^{\mathrm{MRD}}\right|}{\left[\begin{array}{c}
n \\
k-\delta+1
\end{array}\right]_{q} /\left[\begin{array}{c}
k \\
k-\delta+1
\end{array}\right]_{q}}>Q_{\delta}(q) .
$$

The function $Q_{\delta}(q)$ is increasing in $q$ and also in $\delta$. In

\begin{tabular}{|c|c|c|c|c|c|}
\hline$\delta^{\mathrm{q}}$ & 2 & 3 & 4 & 5 & 7 \\
\hline 2 & 0.5776 & 0.8402 & 0.9181 & 0.9504 & 0.9763 \\
\hline 3 & 0.7701 & 0.9452 & 0.9793 & 0.9900 & 0.9966 \\
\hline 4 & 0.8801 & 0.9816 & 0.9948 & 0.9980 & 0.9995 \\
\hline 5 & 0.9388 & 0.9938 & 0.9987 & 0.9996 & 0.9999 \\
\hline
\end{tabular}
Table \, we provide several values of $Q_{\delta}(q)$ for different $q$ and $\delta$. For $q=2$ these values were given in [4].

TABLE I

$Q_{\delta}(q)$

One can verify that for $q$ large enough or for $\delta$ large enough the size of a lifted MRD code approaches the upper bound (2). Thus, an improvement on the lower bound of $\mathcal{A}_{q}(n, 2 \delta, k)$ is mainly important for small minimum distance and small $q$. This will be the line of research in the following sections.

Note, that the lower bound of Lemma 9 is not precise for small values of $k$. But, it is better improved by another construction, the multilevel construction [13]. For example, for $\delta=2$, the lower bound on the ratio between the size of a constant dimension code $\mathbb{C}^{\mathrm{ML}}$ generated by the multilevel construction and the upper bound on $\mathcal{A}_{q}(n, 2 \delta, k)$ given in (2), is presented in Table II] The values in the table are larger than the related values in Table $\llbracket$ In the construction of such a code $\mathbb{C}^{\mathrm{ML}}$ we consider only $\mathbb{C}^{\mathrm{MRD}}$ code and the codewords related to the following three identifying vectors (see [13] or Section IV for the definitions) $\underbrace{11 \ldots 1}_{k-2} 0011 \underbrace{000 \ldots 00}_{n-k-2}, \underbrace{11 \ldots 1}_{k-3} 010101 \underbrace{000 \ldots 00}_{n-k-3}$, and $\underbrace{11 \ldots 1}_{k-2} 000011 \underbrace{000 \ldots 00}_{n-k-4}$, which constitute most of the code. But, since not all identifying vectors were taken in the computations the values in Table II are only lower bounds on the ratio, rather than the exact ratio.

TABLE II

LOWER BOUNDS ON RATIO BETWEEN $\left|\mathbb{C}^{\mathrm{ML}}\right|$ AND THE BOUND IN (2)

\begin{tabular}{|c|c|c|c|c|c|}
\hline $\mathrm{k}^{\mathrm{q}}$ & 2 & 3 & 4 & 5 & 7 \\
\hline 3 & 0.7101 & 0.8678 & 0.9267 & 0.9539 & 0.9771 \\
\hline 4 & 0.6657 & 0.8571 & 0.9231 & 0.9524 & 0.9767 \\
\hline 8 & 0.6274 & 0.8519 & 0.9219 & 0.9520 & 0.9767 \\
\hline 30 & 0.6250 & 0.8518 & 0.9219 & 0.9520 & 0.9767 \\
\hline
\end{tabular}

\section{B. Upper bounds for codes which contain lifted MRD codes}

In this subsection we will derive upper bounds on the size of a constant dimension code which contains the lifted MRD code $\mathbb{C}^{\mathrm{MRD}}$.

Let $\mathbb{T}$ be a subspace transversal design derived from $(n, k, \delta)_{q} \mathbb{C}^{\mathrm{MRD}}$ by Theorem 7 Recall that $\mathcal{L}^{(n, k)}$ is the set of $q^{n}-q^{n-k}$ vectors of length $n$ over $\mathbb{F}_{q}$ in which not all the first $k$ entries are zeroes. Let $\mathcal{L}_{0}^{(n, k)}$ be the set of vectors in $\mathbb{F}_{q}^{n}$ which start with $k$ zeroes. $\mathcal{L}_{0}^{(n, k)}$ is isomorphic to $\mathbb{F}_{q}^{n-k}$, $\left|\mathcal{L}_{0}^{(n, k)}\right|=q^{n-k}$, and $\mathbb{F}_{q}^{n}=\mathcal{L}_{0}^{(n, k)} \cup \mathcal{L}^{(n, k)}$. Note, that $\mathbb{V}_{0}^{(n, k)}$ is the set of one-dimensional subspaces of $\mathcal{G}_{q}(n, 1)$ which contain only vectors from $\mathcal{L}_{0}^{(n, k)}$. A codeword of a constant dimension code, in $\mathcal{G}_{q}(n, k)$, contains one-dimensional subspaces from $\mathcal{G}_{q}(n, 1)=\mathbb{V}_{0}^{(n, k)} \cup \mathbb{V}^{n}$. Let $\mathbb{C}$ be a constant dimension code such that $\mathbb{C}^{\mathrm{MRD}} \subset \mathbb{C}$. Each codeword of $\mathbb{C} \backslash \mathbb{C}^{\mathrm{MRD}}$ contains either at least two points from the same group of $\mathbb{T}$ or only points from $\mathbb{V}_{0}^{(n, k)}$ and hence it contains vectors of $\mathcal{L}_{0}^{(n, k)}$.

Theorem 10: If an $(n, M, 2(k-1), k)_{q}$ code $\mathbb{C}, k \geq 3$, contains an $(n, k, k-1)_{q} \mathbb{C}^{\mathrm{MRD}}$ then $M \leq q^{2(n-k)}+\mathcal{A}_{q}(n-$ $k, 2(k-2), k-1)$.

Proof: Let $\mathbb{T}$ be an $\operatorname{STD}_{q}(2, k, n-k)$ obtained from an $(n, k, k-1)_{q} \mathbb{C}^{\mathrm{MRD}} \subset \mathbb{C}$. Since the minimum distance of $\mathbb{C}$ is $2(k-1)$, it follows that any two codewords of $\mathbb{C}$ intersect in at most one one-dimensional subspace. Hence, each two-dimensional subspace of $\mathbb{F}_{q}^{n}$ is contained in at most one codeword of $\mathbb{C}$. Each two-dimensional subspace $X$ of $\mathbb{F}_{q}^{n}$, such that $X=\langle\{v, u\}\rangle, v \in \mathbb{V}_{A}^{(n, k)}, u \in \mathbb{V}_{B}^{(n, k)}$, where $A \neq B, A, B \in \mathcal{G}_{q}(k, 1)$, is contained in a codeword of $\mathbb{C}^{\mathrm{MRD}}$ by Corollary 4 (or by Theorem 7). Hence, each codeword $X \in \mathbb{C} \backslash \mathbb{C}^{\mathrm{MRD}}$ either contains only points from $\mathbb{V}_{0}^{(n, k)}$ or contains points from $\mathbb{V}_{0}^{(n, k)}$ and points from $\mathbb{V}_{A}^{(n, k)}$, for some $A \in \mathcal{G}_{q}(k, 1)$. Clearly, $\operatorname{dim}\left(X \cap \mathcal{L}_{0}^{(n, k)}\right)=k$ in the first case and $\operatorname{dim}\left(X \cap \mathcal{L}_{0}^{(n, k)}\right)=k-1$ in the second case. Since $k \geq 3$ and two codewords of $\mathbb{C}$ intersect in at most a one-dimensional subspace, it follows that each $(k-1)$-dimensional subspace of $\mathcal{L}_{0}^{(n, k)}$ can be contained only 
in one codeword. Moreover, since the minimum distance of the code is $2(k-1)$, it follows that if $X_{1}, X_{2} \in \mathbb{C} \backslash \mathbb{C}^{\mathrm{MRD}}$ and $\operatorname{dim}\left(X_{1} \cap \mathcal{L}_{0}^{(n, k)}\right)=\operatorname{dim}\left(X_{2} \cap \mathcal{L}_{0}^{(n, k)}\right)=k-1$ then $d_{S}\left(X_{1} \cap \mathcal{L}_{0}^{(n, k)}, X_{2} \cap \mathcal{L}_{0}^{(n, k)}\right) \geq 2(k-2)$. Therefore, $\mathbb{C}^{\prime} \stackrel{\text { def }}{=}\left\{X \cap \mathcal{L}_{0}^{(n, k)}: X \in \mathbb{C} \backslash \mathbb{C}^{\mathrm{MRD}}, \operatorname{dim}\left(X \cap \mathcal{L}_{0}^{(n, k)}\right)=k-1\right\}$ is an $\left(n-k, M^{\prime}, 2(k-2), k-1\right)_{q}$ code. Let $\mathbb{S}$ be the set of codewords in $\mathbb{C} \backslash \mathbb{C}^{\mathrm{MRD}}$ such that $\operatorname{dim}\left(X \cap \mathcal{L}_{0}\right)=k$. For each $X \in \mathbb{S}$ let $\tilde{X}$ be an arbitrary $(k-1)$-dimensional subspace of $X$, and let $\mathbb{S}^{\prime} \stackrel{\text { def }}{=}\{\tilde{X}: X \in \mathbb{S}\}$ (note that $\left|\mathbb{S}^{\prime}\right|=|\mathbb{S}|$ ). Since $d_{S}\left(\mathbb{C}^{\prime}\right) \geq 2(k-2), k \geq 3$, and each two codewords of $\mathbb{C}$ intersect in at most a one-dimensional subspace, it follows that the code $\mathbb{C}^{\prime} \cup \mathbb{S}^{\prime}$ is an $\left(n-k, M^{\prime \prime}, 2(k-2), k-1\right)_{q}$ code. This implies the result of the theorem.

Theorem 11: If an $(n, M, 2 k, 2 k)_{q}$ code $\mathbb{C}$ contains an $(n, 2 k, k)_{q} \quad \mathbb{C}^{\mathrm{MRD}}$ then $M \leq q^{(n-2 k)(k+1)}+$ $\left[\begin{array}{c}n-2 k \\ k\end{array}\right]_{q} \frac{q^{n}-q^{n-2 k}}{q^{2 k}-q^{k}}+\mathcal{A}_{q}(n-2 k, 2 k, 2 k)$.

Proof: Let $\mathbb{T}$ be an $\operatorname{STD}_{q}(k+1,2 k, n-2 k)$ obtained from an $(n, 2 k, k)_{q} \mathbb{C}^{\mathrm{MRD}} \subset \mathbb{C}$. Since the minimum distance of $\mathbb{C}$ is $2 k$, it follows that any two codewords of $\mathbb{C}$ intersect in at most a $k$-dimensional subspace. Hence, each $(k+1)$ dimensional subspace of $\mathbb{F}_{q}^{n}$ is contained in at most one codeword of $\mathbb{C}$. Each $(k+1)$-dimensional subspace $Y$ of $\mathbb{F}_{q}^{n}$, such that $Y=\left\langle\left\{v_{1}, \ldots, v_{k}, v_{k+1}\right\}\right\rangle, v_{i} \in \mathbb{V}_{A_{i}}^{(n, 2 k)}$, where $A_{i} \neq A_{j}$, for $i \neq j$, and $A_{i} \in \mathcal{G}_{q}(2 k, 1), 1 \leq i \leq k+1$, is contained in a codeword of $\mathbb{C}^{\mathrm{MRD}}$ by Theorem 7 Hence, each codeword $X \in \mathbb{C} \backslash \mathbb{C}^{M R D}$ has a nonempty intersection with exactly $\frac{q^{k-\tau}-1}{q-1}$ groups of $\mathbb{T}$, for some $0 \leq \tau \leq k$ and therefore $\operatorname{dim}\left(X \cap \mathcal{L}_{0}^{(n, 2 k)}\right)=k+\tau$. Let $\mathbb{S}_{\tau}$ be the set of codewords defined by, $X \in \mathbb{S}_{\tau}$ if $\operatorname{dim}\left(X \cap \mathcal{L}_{0}^{(n, 2 k)}\right)=k+\tau$.

The set $\mathbb{S}_{k}$ forms an $\left(n-2 k, M^{\prime}, 2 k, 2 k\right)_{q}$ code and hence $\left|\mathbb{S}_{k}\right| \leq \mathcal{A}_{q}(n-2 k, 2 k, 2 k)$.

Let $Y$ be a $k$-dimensional subspace of $\mathcal{L}_{0}^{(n, 2 k)}$. If $X_{1}$ and $X_{2}$ are two codewords which contain $Y$ then $Y=X_{1} \cap X_{2}$. Let $N_{\tau, Y}$ be the number of codewords from $\mathbb{S}_{\tau}$ which contain $Y$. Clearly, for each $\tau, 0 \leq \tau \leq k$, we have

$$
\sum_{Y \in \mathcal{G}_{q}(n-2 k, k)} N_{\tau, Y}=\left|\mathbb{S}_{\tau}\right|\left[\begin{array}{c}
k+\tau \\
k
\end{array}\right]_{q}
$$

There are $\frac{q^{n}-q^{n-2 k}}{q-1}$ points in $\mathbb{V}^{n}$ and each $X \in \mathbb{S}_{\tau}$ contains exactly $\frac{q^{2 k}-q^{k+\tau}}{q-1}$ points from $\mathbb{V}^{n}$. Hence, each $k$-dimensional subspace $Y$ of $\mathcal{L}_{0}^{(n, 2 k)}$ can be a subspace of at most $\frac{q^{n}-q^{n-2 k}-\sum_{\tau=1}^{k-1} N_{\tau, Y}\left(q^{2 k}-q^{k+\tau}\right)}{q^{2 k}-q^{k}}$ codewords of $\mathbb{S}_{0}$.

Therefore,

$$
\begin{gathered}
|\mathbb{C}| \leq q^{(n-2 k)(k+1)}+\sum_{\tau=1}^{k}\left|\mathbb{S}_{\tau}\right| \\
+\sum_{Y \in \mathcal{G}_{q}(n-2 k, k)} \frac{q^{n}-q^{n-2 k}-\sum_{\tau=1}^{k-1} N_{\tau, Y}\left(q^{2 k}-q^{k+\tau}\right)}{q^{2 k}-q^{k}} \\
=q^{(n-2 k)(k+1)}+\sum_{\tau=1}^{k}\left|\mathbb{S}_{\tau}\right|+\left(\left[\begin{array}{c}
n-2 k \\
k
\end{array}\right] \frac{q^{n}-q^{n-2 k}}{q^{2 k}-q^{k}}\right.
\end{gathered}
$$

$$
\left.-\sum_{\tau=1}^{k-1}\left|\mathbb{S}_{\tau}\right|\left[\begin{array}{c}
k+\tau \\
k
\end{array}\right]_{q} \frac{q^{2 k}-q^{k+\tau}}{q^{2 k}-q^{k}}\right),
$$

where the equality is derived from (3).

One can easily verify that $\left[\begin{array}{c}k+\tau \\ k\end{array}\right]_{q} \frac{q^{2 k}-q^{k+\tau}}{q^{2 k}-q^{k}} \geq 1$ for $1 \leq \tau \leq k-1$; recall also that $\left|\mathbb{S}_{k}\right| \leq \mathcal{A}_{q}(n-2 k, 2 k, 2 k)$; thus we have

$$
|\mathbb{C}| \leq q^{(n-2 k)(k+1)}+\left[\begin{array}{c}
n-2 k \\
k
\end{array}\right]_{q} \frac{q^{n}-q^{n-2 k}}{q^{2 k}-q^{k}}+\mathcal{A}_{q}(n-2 k, 2 k, 2 k) .
$$

\section{Constructions for $(n, M, 4,3)_{q}$ CODES}

In this section we discuss and present a construction of codes which contain an $(n, k, \delta) \mathbb{C}^{\mathrm{MRD}}$ and attain the bound of Theorem 10. Such a construction is presented only for $k=3$ and $q$ large enough. If $q$ is not large enough then codes obtained by a modification of this construction almost attain the bound. In any case the codes obtained in this section are the largest ones known for $k=3$ and $\delta=2$.

For $k=3$, the upper bound of Theorem 10 on the size of a code which contains an $(n, 3,2)_{q} \mathbb{C}^{\mathrm{MRD}}$ is $q^{2(n-3)}+\left[\begin{array}{c}n-3 \\ 2\end{array}\right]_{q}$. The construction which follows is inspired by the construction methods described in [13] and [48]. The construction is based on representation of subspaces by Ferrers diagrams, optimal rank-metric codes, pending dots, and one-factorization of the complete graph. The definitions and results of the first subsection are taken from [13], [31], and [48].

\section{A. Preliminaries for the construction}

1) Representation of subspaces: For each $X \in \mathcal{G}_{q}(n, k)$ represented by the generator matrix in reduced row echelon form, denoted by $\operatorname{RE}(X)$, we associate a binary vector of length $n$ and weight $k, v(X)$, called the identifying vector of $X$, where the ones in $v(X)$ are exactly in the positions where $\operatorname{RE}(X)$ has the leading coefficients (the pivots). All the binary vectors of length $n$ and weight $k$ can be considered as the identifying vectors of all the subspaces in $\mathcal{G}_{q}(n, k)$. These $\left(\begin{array}{l}n \\ k\end{array}\right)$ vectors partition $\mathcal{G}_{q}(n, k)$ into the $\left(\begin{array}{l}n \\ k\end{array}\right)$ different classes, where each class consists of all subspaces in $\mathcal{G}_{q}(n, k)$ with the same identifying vector.

The Ferrers tableaux form of a subspace $X$, denoted by $\mathcal{F}(X)$, is obtained from $\operatorname{RE}(X)$ first by removing from each row of $\operatorname{RE}(X)$ the zeroes to the left of the leading coefficient; and after that removing the columns which contain the leading coefficients. All the remaining entries are shifted to the right. The Ferrers diagram of $X$, denoted by $\mathcal{F}_{X}$, is obtained from $\mathcal{F}(X)$ by replacing the entries of $\mathcal{F}(X)$ with dots. Given $\mathcal{F}(X)$, the unique corresponding subspace $X \in \mathcal{G}_{q}(n, k)$ can be easily found.

Example 3: Let $X$ be the subspace in $\mathcal{G}_{2}(7,3)$ with the following generator matrix in reduced row echelon form:

$$
\operatorname{RE}(X)=\left(\begin{array}{lllllll}
\mathbf{1} & 0 & 0 & 0 & 1 & 1 & 0 \\
0 & 0 & \mathbf{1} & 0 & 1 & 0 & 1 \\
0 & 0 & 0 & \mathbf{1} & 0 & 1 & 1
\end{array}\right)
$$


Its identifying vector is $v(X)=1011000$, and its Ferrers tableaux form and Ferrers diagram are given by

$\begin{array}{rrrrrrrrr}0 & 1 & 1 & 0 \\ & 1 & 0 & 1 \\ & 0 & 1 & 1\end{array} \quad$ and $\quad \begin{array}{llll}\bullet & \bullet & \bullet & \bullet\end{array}$

2) Lifted Ferrers diagram rank-metric codes: Let $\mathcal{F}$ be a Ferrers diagram with $k$ dots in the rightmost column and $\ell$ dots in the top row. A code $\mathcal{C}_{\mathcal{F}}$ is an $[\mathcal{F}, \varrho, \delta]$ Ferrers diagram rank-metric code if all codewords of $\mathcal{C}_{\mathcal{F}}$ are $k \times \ell$ matrices in which all entries not in $\mathcal{F}$ are zeroes, it forms a rank-metric code with dimension $\varrho$ and minimum rank distance $\delta$. The following result is the direct consequence from Theorem 1 in [13].

Lemma 12: Let $n \geq 8, k=3, \delta=2$, and let $v$ be an identifying vector, of length $n$ and weight three, in which the leftmost one appears in one of the first three entries. Let $\mathcal{F}$ be the corresponding Ferrers diagram and $[\mathcal{F}, \varrho, 2]$ be a Ferrers diagram rank-metric code. Then $\varrho$ is at most the number of dots in $\mathcal{F}$, which are not contained in its first row.

A code which attains the bound of Lemma 12 will be called a Ferrers diagram MRD code. A construction for such codes can be found in [13].

For a codeword $A \in \mathcal{C}_{\mathcal{F}} \subset \mathbb{F}_{q}^{k \times(n-k)}$, let $A_{\mathcal{F}}$ denotes the part of $A$ related to the entries of $\mathcal{F}$ in $A$. Given a Ferrers diagram MRD code $\mathcal{C}_{\mathcal{F}}$, a lifted Ferrers diagram MRD code $\mathbb{C}_{\mathcal{F}}$ is defined as follows:

$$
\mathbb{C}_{\mathcal{F}}=\left\{X \in \mathcal{G}_{q}(n, k): \mathcal{F}(X)=A_{\mathcal{F}}, A \in \mathcal{C}_{\mathcal{F}}\right\} .
$$

This definition is the generalization of the definition of a lifted MRD code. The following lemma [13] is the generalization of the result given in Theorem 1 .

Lemma 13: If $\mathcal{C}_{\mathcal{F}} \subset \mathbb{F}_{q}^{k \times(n-k)}$ is an $[\mathcal{F}, \varrho, \delta]$ Ferrers diagram rank-metric code, then its lifted code $\mathbb{C}_{\mathcal{F}}$ is an $\left(n, q^{\varrho}, 2 \delta, k\right)_{q}$ constant dimension code.

3) The multilevel construction and pending dots: It was proved in [13] that for any two subspaces $X, Y \in \mathcal{G}_{q}(n, k)$ we have $d_{S}(X, Y) \geq d_{H}(v(X), v(Y))$, where $d_{H}$ denotes the Hamming distance; and if $v(X)=v(Y)$ then $d_{S}(X, Y)=$ $2 d_{R}(\operatorname{RE}(X), \operatorname{RE}(Y))$. These properties of the subspace distance were used in [13] to present a multilevel construction, for a constant dimension code $\mathbb{C}$. In this construction, first a binary constant weight code $C$ of length $n$, weight $k$, and minimum Hamming distance $2 \delta$ is chosen. The codewords of $C$ will serve as the identifying vectors for $\mathbb{C}$. For each identifying vector a corresponding lifted Ferrers diagram MRD code with minimum rank distance $\delta$ is constructed. The union of these lifted Ferrers diagram MRD codes is an $(n, M, 2 \delta, k)_{q}$ code.

In the construction which follows, for $\delta=2$, we also use a multilevel method, i.e., we first choose a binary constant weight code $C$ of length $n$, weight $k=3$, and minimum Hamming distance $2 \delta-2=2$. For each codeword in $C$ a corresponding lifted Ferrers diagram MRD code is constructed. However, since for some pairs of identifying vectors the Hamming distance is 2, we need to use appropriate lifted Ferrers diagram MRD codes to make sure that the final subspace distance of the code will be 4 . For this purpose we use a method based on pending dots in a Ferrers diagram [48].

The pending dots of a Ferrers diagram $\mathcal{F}$ are the leftmost dots in the first row of $\mathcal{F}$ whose removal has no impact on the size of the corresponding Ferrers diagram rank-metric code. The following lemma follows from [48].

Lemma 14: [48] Let $X$ and $Y$ be two subspaces in $\mathcal{G}_{q}(n, k)$ with $d_{H}(v(X), v(Y))=2 \delta-2$, such that the leftmost one of $v(X)$ is in the same position as the leftmost one of $v(Y)$. Let $P_{X}$ and $P_{Y}$ be the sets of pending dots of $X$ and $Y$, respectively. If $P_{X} \cap P_{Y} \neq \varnothing$ and the entries in $P_{X} \cap P_{Y}$ (of their Ferrers tableaux forms) are assigned with different values in at least one position, then $d_{S}(X, Y) \geq 2 \delta$.

Example 4: Let $X$ and $Y$ be subspaces in $\mathcal{G}_{q}(8,3)$ which are given by the following generator matrices:

$$
\begin{aligned}
& \mathrm{RE}(X)=\left(\begin{array}{cccccccc}
1 & (0) & 0 & 0 & v_{1} & v_{2} & 0 & v_{3} \\
0 & 0 & 0 & 1 & v_{4} & v_{5} & 0 & v_{6} \\
0 & 0 & 0 & 0 & 0 & 0 & 1 & v_{7}
\end{array}\right) \\
& \operatorname{RE}(Y)=\left(\begin{array}{cccccccc}
1 & 0 & 1 & v_{1}^{\prime} & 0 & v_{2}^{\prime} & 0 & v_{3}^{\prime} \\
0 & 0 & 0 & 0 & 1 & v_{4}^{\prime} & 0 & v_{5}^{\prime} \\
0 & 0 & 0 & 0 & 0 & 0 & 1 & v_{6}^{\prime}
\end{array}\right)
\end{aligned}
$$

where $v_{i}, v_{i}^{\prime} \in \mathbb{F}_{q}$, and the pending dots are emphasized by circles. Their identifying vectors are $v(X)=10010010$ and $v(Y)=10001010$. Clearly, $d_{H}(v(X), v(Y))=2$, while $d_{S}(X, Y)=4$.

4) One-factorization of complete graphs: A matching in a graph $G$ is a set of pairwise disjoint edges of $G$. A one-factor is a matching such that every vertex of $G$ occurs in exactly one edge of the matching. A partition of the edge set in $G$ into one-factors is called a one-factorization. Let $K_{n}$ be a complete graph with $n$ vertices. The following lemma is a well known result [31, p. 476].

Lemma 15: $K_{2 n}$ has a one-factorization for all $n$.

A near-one-factor in $K_{2 n-1}$ is a matching with $n-1$ edges which contain all but one vertex. A set of near-one-factors which contains each edge in $K_{2 n-1}$ precisely once is called a near-one-factorization. The following corollary is the direct consequence from Lemma 15.

Corollary 5: $K_{2 n-1}$ has a near-one-factorization for all $n$.

Corollary 6: Let $D$ be a set of all binary vectors of length $m$ and weight 2 .

- If $m$ is even, $D$ can be partitioned into $m-1$ classes, each one has $\frac{m}{2}$ vectors with pairwise disjoint positions of ones:

- If $m$ is odd, $D$ can be partitioned into $m$ classes, each one has $\frac{m-1}{2}$ vectors with pairwise disjoint positions of ones.

\section{B. The first construction}

Construction I: Let $n \geq 8$ and $q^{2}+q+1 \geq n-4$ for odd $n$ (or $q^{2}+q+1 \geq n-3$ for even $n$ ). 
1) Identifying vectors: The identifying vector $v_{0}=$ $11100 \ldots 0$ corresponds to the lifted MRD code $\mathbb{C}^{\mathrm{MRD}}$. The other identifying vectors are of the form $x \| y$, where $x$ is of length 3 and weight one, and $y$ is of length $n-3$ and weight two. We use all the $\left(\begin{array}{c}n-3 \\ 2\end{array}\right)$ vectors of weight two in the last $n-3$ coordinates of the identifying vectors. By Corollary 6 there is a partition of the set of vectors of length $n-3$ and weight 2 into $s=n-4$ classes if $n-3$ is even (or into $s=n-3$ classes if $n-3$ is odd), $P_{1}, P_{2}, \ldots, P_{s}$. We define

$$
\begin{gathered}
\mathcal{A}_{1}=\left\{(001) \| y: y \in P_{1}\right\}, \\
\mathcal{A}_{2}=\left\{(010) \| y: y \in P_{i}, 2 \leq i \leq \min \{q+1, s\}\right\}, \\
\mathcal{A}_{3}=\left\{\begin{array}{cc}
\left\{(100) \| y: y \in P_{i}, q+2 \leq i \leq s\right\} & \text { if } s>q+1 \\
\varnothing & \text { if } s \leq q+1
\end{array}\right.
\end{gathered}
$$

2) Ferrers tableaux forms and pending dots: All the Ferrers diagrams which correspond to the identifying vectors from $\mathcal{A}_{2}$ have one common pending dot in the first entry of the first row. We assign the same value of $\mathbb{F}_{q}$ in this entry of the Ferrers tableaux form for each vector in the same class. Two subspaces with identifying vectors from different classes of $\mathcal{A}_{2}$ have different values in the entry of this pending dot. This is possible since the number of classes in $\mathcal{A}_{2}$ is at most $q$. On the remaining dots of Ferrers diagrams we construct Ferrers diagram MRD codes and lift them.

Similarly, all the Ferrers diagrams which correspond to the identifying vectors from $\mathcal{A}_{3}$, have two common pending dots in the first two entries of the first row. We assign the same value of $\mathbb{F}_{q}$ in these two entries in the Ferrers tableaux form for each vector in the same class. Two subspaces with identifying vectors from different classes of $\mathcal{A}_{3}$ have different values in at least one of these two entries. This is possible since the number of classes in $\mathcal{A}_{3}$ is at most $q^{2}$. On the remaining dots of Ferrers diagrams we construct Ferrers diagram MRD codes and lift them.

Finally, we lift Ferrers diagrams MRD codes which correspond to the identifying vectors of $\mathcal{A}_{1}$.

3) The code: Our code $\mathbb{C}$ is a union of $\mathbb{C}^{\mathrm{MRD}}$ and the lifted codes corresponding to the identifying vectors in $\mathcal{A}_{1}$, $\mathcal{A}_{2}$, and $\mathcal{A}_{3}$.

Example 5: For $n=8$, there are $\left(\begin{array}{l}5 \\ 2\end{array}\right)$ different binary vectors of length $8-3=5$ and weight 2 . We partition these vectors into five disjoint classes $P_{1}=\{(11000),(00110)\}$, $P_{2}=\{(10100),(01001)\}, P_{3}=\{(10010),(00101)\}, P_{4}=$ $\{(10001),(01010)\}, P_{5}=\{(01100),(00011)\}$. The identifying vectors of the code, besides $v_{0}=11100000$, are partitioned into three sets,

$$
\begin{gathered}
\mathcal{A}_{1}=\{(00111000),(00100110)\}, \\
\mathcal{A}_{2}=\{(01010100),(01001001),(01010010),(01000101)\}, \\
\mathcal{A}_{3}=\{(10010001),(10001010),(10001100),(10000011)\} .
\end{gathered}
$$

To demonstrate the idea of the construction we will only consider the set $\mathcal{A}_{2}$. The generator matrices in reduced row echelon form of the codewords with identifying vectors from $\mathcal{A}_{2}$ are of four different types:

$$
\begin{aligned}
& \left(\begin{array}{cccccccc}
0 & 1 & v_{1}^{1} & 0 & v_{2}^{1} & 0 & v_{3}^{2} & v_{4}^{1} \\
0 & 0 & 0 & 1 & v_{5}^{1} & 0 & v_{6}^{1} & v_{7}^{1} \\
0 & 0 & 0 & 0 & 0 & 1 & v_{8}^{1} & v_{9}^{1}
\end{array}\right), \\
& \left(\begin{array}{ccccccccc}
0 & 1 & v_{1}^{2} & v_{2}^{2} & 0 & v_{3}^{2} & v_{4}^{2} & 0 \\
0 & 0 & 0 & 0 & 1 & v_{5}^{2} & v_{6}^{2} & 0 \\
0 & 0 & 0 & 0 & 0 & 0 & 0 & 1
\end{array}\right), \\
& \left(\begin{array}{ccccccccc}
0 & 1 & v_{1}^{3} & 0 & v_{2}^{3} & v_{3}^{3} & 0 & v_{4}^{3} \\
0 & 0 & 0 & 1 & v_{5}^{3} & v_{6}^{3} & 0 & v_{7}^{3} \\
0 & 0 & 0 & 0 & 0 & 0 & 1 & v_{8}^{3}
\end{array}\right), \\
& \left(\begin{array}{ccccccccc}
0 & 1 & v_{1}^{4} & v_{2}^{4} & v_{3}^{4} & 0 & v_{4}^{4} & 0 \\
0 & 0 & 0 & 0 & 0 & 1 & v_{5}^{4} & 0 \\
0 & 0 & 0 & 0 & 0 & 0 & 0 & 1
\end{array}\right),
\end{aligned}
$$

where all the $v_{i}^{j}$,s are elements from $\mathbb{F}_{q}$. The suffixes (last $n-3$ coordinates) of the identifying vectors of the first two generator matrices belong to $P_{2}$, and of the last two matrices to $P_{3}$. All these matrices have the same pending dot in the place of $v_{1}^{i}, 1 \leq i \leq 4$. Then we assign 0 in this place for the two first matrices and 1 in this place for the two last matrices:

$$
\begin{aligned}
& \left(\begin{array}{cccccccc}
0 & 1 & \mathbf{0} & 0 & v_{2}^{1} & 0 & v_{3}^{1} & v_{4}^{1} \\
0 & 0 & 0 & 1 & v_{5}^{1} & 0 & v_{6}^{1} & v_{7}^{1} \\
0 & 0 & 0 & 0 & 0 & 1 & v_{8}^{1} & v_{9}^{1}
\end{array}\right), \\
& \left(\begin{array}{ccccccccc}
0 & 1 & \mathbf{0} & v_{2}^{2} & 0 & v_{3}^{2} & v_{4}^{2} & 0 \\
0 & 0 & 0 & 0 & 1 & v_{5}^{2} & v_{6}^{2} & 0 \\
0 & 0 & 0 & 0 & 0 & 0 & 0 & 1
\end{array}\right), \\
& \left(\begin{array}{ccccccccc}
0 & 1 & \mathbf{1} & 0 & v_{2}^{3} & v_{3}^{3} & 0 & v_{4}^{3} \\
0 & 0 & 0 & 1 & v_{5}^{3} & v_{6}^{3} & 0 & v_{7}^{3} \\
0 & 0 & 0 & 0 & 0 & 0 & 1 & v_{8}^{3}
\end{array}\right), \\
& \left(\begin{array}{cccccccc}
0 & 1 & \mathbf{1} & v_{2}^{4} & v_{3}^{4} & 0 & v_{4}^{4} & 0 \\
0 & 0 & 0 & 0 & 0 & 1 & v_{5}^{4} & 0 \\
0 & 0 & 0 & 0 & 0 & 0 & 0 & 1
\end{array}\right) .
\end{aligned}
$$

4) Analysis of the construction:

Theorem 16: For $q$ satisfying $q^{2}+q+1 \geq s$, where

$$
s=\left\{\begin{array}{cc}
n-4, & n \text { is odd } \\
n-3, & n \text { is even }
\end{array},\right.
$$

the code $\mathbb{C}$ obtained in Construction I attains the bound of Theorem 10.

Proof: First, we prove that the minimum subspace distance of $\mathbb{C}$ is 4 . Let $X, Y \in \mathbb{C}, X \neq Y$. We distinguish between three cases:

- Case 1: If $X, Y \in \mathbb{C}^{\mathrm{MRD}}$ then $d_{S}(X, Y) \geq 4$ since the minimum distance of the $(n, 3,2)_{q} \mathbb{C}^{\mathrm{MRD}}$ is 4 .

- Case 2: If $X \in \mathbb{C}^{\mathrm{MRD}}$ and $Y \in \mathbb{C} \backslash \mathbb{C}^{\mathrm{MRD}}$ then $d_{S}(X, Y) \geq d_{H}(v(X), v(Y)) \geq 4$.

- Case 3: Assume $X, Y \in \mathbb{C} \backslash \mathbb{C}^{\mathrm{MRD}}$. If $v(X) \in \mathcal{A}_{i}, v(Y) \in \mathcal{A}_{j}, i \neq j$, then clearly $d_{S}(X, Y) \geq d_{H}(v(X), v(Y)) \geq 4$.

If $v(X), v(Y) \in \mathcal{A}_{i}$, i.e., $X$ and $Y$ have identifying vectors $v(X)=z\|w, v(Y)=z\| w^{\prime}$, where $z$ is of length 3 , we distinguish between two additional cases: 
- $w, w^{\prime} \in P_{i}, 1 \leq i \leq s$. In this case $d_{H}(v(X), v(Y))=4$ which implies $d_{S}(X, Y) \geq 4$.

- $w \in F_{i}, w^{\prime} \in F_{j}, i \neq j$. If $d_{H}(v(X), v(Y))=4$ then $d_{S}(X, Y) \geq 4$. If $d_{H}(v(X), v(Y))=2$ then by Lemma 14 we have that $d_{S}(X, Y) \geq 4$.

Next, we calculate the size of $\mathbb{C}$. Recall, that the identifying vectors are partitioned into $s$ classes. Note that since $q^{2}+q+1 \geq s$, it follows that each one of the $\left(\begin{array}{c}n-3 \\ 2\end{array}\right)$ vectors of weight 2 and length $n-3$ is taken as the suffix of some identifying vector. Each such suffix (of length $n-3$ and weight 2) is the identifying vector of a subspace in $\mathcal{G}_{q}(n-3,2)$. By Lemma 12 each such subspace in $\mathcal{G}_{q}(n-3,2)$ is contained in exactly one codeword (since the first row of the generator matrix of the 3-dimensional subspace is omitted by the lemma for the bound on $\varrho$ ). The size of $\mathbb{C}^{\mathrm{MRD}}$ is $q^{2(n-3)}$ and the size of $\mathcal{G}_{q}(n-3,2)$ is $\left[\begin{array}{c}n-3 \\ 2\end{array}\right]_{q}$. Hence, the size of $\mathbb{C}$ is $q^{2(n-3)}+\left[\begin{array}{c}n-3 \\ 2\end{array}\right]_{q}$. Theorem 10 implies that for $(n, M, 4,3)_{q}$ code $\mathbb{C}$, which contains an $(n, 3,2)_{q} \mathbb{C}^{\mathrm{MRD}}$ we have $M \leq q^{2(n-3)}+\mathcal{A}_{q}(n-3,2,2)=q^{2(n-3)}+\left[\begin{array}{c}n-3 \\ 2\end{array}\right]_{q}$.

Remark 3: A $(6, M, 4,3)_{q}$ code whose size attains the upper bound of Theorem [10] was constructed in [13] and a $(7, M, 4,3)_{q}$ code whose size attains this bound was constructed in [48].

\section{The second construction}

For small alphabets Construction I is modified as follows.

Construction II: Let $n \geq 8$ and $q^{2}+q+1<n-4$ for odd $n$ (or $q^{2}+q+1<n-3$ for even $n$ ).

The identifying vector $v_{0}=11100 \ldots 0$ corresponds to the lifted MRD code $\mathbb{C}^{\mathrm{MRD}}$. Let $\alpha=\left\lfloor\frac{n-3}{q^{2}+q+2}\right\rfloor$ and $r=n-3-$ $\alpha\left(q^{2}+q+2\right)$. For each other identifying vector, we partition the last $n-3$ coordinates into $\alpha$ or $\alpha+1$ sets, where each one of the first $\alpha$ sets consists of $q^{2}+q+2$ consecutive coordinates and the last set (which exists if $r>0$ ) consists of $r<q^{2}+q+2$ consecutive coordinates. Since $q^{2}+q+2$ is always an even integer, it follows from Corollary 6 that there is a partition of vectors of length $q^{2}+q+2$ and weight 2 , corresponding to the $i$ th set, $1 \leq i \leq \alpha$, into $q^{2}+q+1$ classes $P_{1}^{i}, P_{2}^{i}, \ldots, P_{q^{2}+q+1}^{i}$. We define $Y_{1}^{i}=\left\{0^{(i-1)\left(q^{2}+q+2\right)}\|y\| 0^{n-3-i\left(q^{2}+q+2\right)}: y \in\right.$ $\left.P_{1}^{i}\right\}, Y_{2}^{i}=\left\{0^{(i-1)\left(q^{2}+q+2\right)}\|y\| 0^{n-3-i\left(q^{2}+q+2\right)}: y \in P_{j}^{i}, 2 \leq\right.$ $j \leq q+1\}$, and $Y_{3}^{i}=\left\{0^{(i-1)\left(q^{2}+q+2\right)}\|y\| 0^{n-3-i\left(q^{2}+q+2\right)}\right.$ : $\left.y \in P_{j}^{i}, q+2 \leq j \leq q^{2}+q+1\right\}$, where $0^{\ell}$ denotes the zeroes vector of length $\ell$. Let

$$
\begin{aligned}
& \mathcal{A}_{1}^{i}=\left\{(001) \| y: y \in Y_{1}^{i}\right\}, 1 \leq i \leq \alpha, \\
& \mathcal{A}_{2}^{i}=\left\{(010) \| y: y \in Y_{2}^{i}\right\}, 1 \leq i \leq \alpha, \\
& \mathcal{A}_{3}^{i}=\left\{(100) \| y: y \in Y_{3}^{i}\right\}, 1 \leq i \leq \alpha .
\end{aligned}
$$

The identifying vectors (excluding $v_{0}$ ), of the code that we construct, are partitioned into the following three sets:

$$
\mathcal{A}_{1}=\cup_{i=1}^{\alpha} \mathcal{A}_{1}^{i}, \mathcal{A}_{2}=\cup_{i=1}^{\alpha} \mathcal{A}_{2}^{i}, \mathcal{A}_{3}=\cup_{i=1}^{\alpha} \mathcal{A}_{3}^{i} .
$$

As in Construction I, we construct a lifted Ferrers diagram MRD code for each identifying vector, by using pending dots. Our code $\mathbb{C}$ is a union of $\mathbb{C}^{\mathrm{MRD}}$ and the lifted codes corresponding to the identifying vectors in $\mathcal{A}_{1}, \mathcal{A}_{2}$, and $\mathcal{A}_{3}$.

Remark 4: The identifying vectors with two ones in the last $r$ entries can be also used in Construction II, but their contribution to the final code is minor.

In a similar way to the proof of Theorem 16 one can prove the following theorem, based on the fact that the size of the lifted Ferrers diagram MRD code obtained from the identifying vectors in $\mathcal{A}_{1}^{i} \cup \mathcal{A}_{2}^{i} \cup \mathcal{A}_{3}^{i}, 1 \leq i \leq \alpha$, is $\left[\begin{array}{c}q^{2}+q+2 \\ 2\end{array}\right]_{q} q^{2\left(n-3-\left(q^{2}+q+2\right) i\right)}$.

Theorem 17: For $q$ satisfying $q^{2}+q+1<s$, where

$$
s=\left\{\begin{array}{ll}
n-4, & n \text { is odd } \\
n-3, & n \text { is even }
\end{array},\right.
$$

Construction II generates an $(n, M, 4,3)_{q}$ constant dimension code with $M=q^{2(n-3)}+$ $\sum_{i=1}^{\alpha}\left[\begin{array}{c}q^{2}+q+2 \\ 2\end{array}\right]_{q} q^{2\left(n-3-\left(q^{2}+q+2\right) i\right)}$, which contains an $(n, 3,2)_{q} \mathbb{C}^{\mathrm{MRD}}$.

For all admissible values of $n$, the ratio $(|\mathbb{C}|-$ $\left.\left|\mathbb{C}^{\mathrm{MRD}}\right|\right) /\left[\begin{array}{c}n-3 \\ 2\end{array}\right]_{q}$, for the code $\mathbb{C}$ generated by Construction II, is greater than 0.988 for $q=2$ and 0.999 for $q>2$. Hence, the code almost attains the bound of Theorem 10.

In the following table we compare the size of codes obtained by Constructions I and II (denoted by $\mathbb{C}_{n e w}$ ) with the size of the largest previously known codes (denoted by $\mathbb{C}_{\text {old }}$ ) and with the upper bound (2) (for $k=3$ ).

\begin{tabular}{|c|c|c|c|c|}
\hline$q$ & $n$ & $\left|\mathbb{C}_{\text {old }}\right|$ & $\left|\mathbb{C}_{\text {new }}\right|$ & upper bound [2] \\
\hline \hline 2 & 13 & $1192587 \llbracket 13 \rrbracket$ & 1221296 & 1597245 \\
\hline 2 & 14 & $4770411 \llbracket 13 \rrbracket$ & 4885184 & 6390150 \\
\hline 5 & 9 & $244644376 \llbracket 13 \rrbracket$ & 244649056 & 256363276 \\
\hline
\end{tabular}

The new ratio between the new best lower bound and the

\begin{tabular}{|c|c|c|c|c|c|}
\hline $\mathrm{k}^{\mathrm{q}}$ & 2 & 3 & 4 & 5 & 7 \\
\hline 3 & 0.7657 & 0.8738 & 0.928 & 0.9543 & 0.9772 \\
\hline
\end{tabular}
upper bound (2) with $k=3$ and $\delta=2$, is presented in Table III. One should compare it with Table II

TABLE III

The Ratio between $\left|\mathbb{C}_{n e w}\right|$ AND the bound in 2]

\section{Construction For $(8, M, 4,4)_{q}$ CODES}

In this section we introduce a construction of $(8, M, 4,4)_{q}$ codes which attain the upper bound of Theorem 11, and are the largest codes with these parameters. This construction is based on 2-parallelism of subspaces in $\mathcal{G}_{q}(4,2)$.

A $k$-spread in $\mathcal{G}_{q}(n, k)$ is a set of $k$-dimensional subspaces which partition $\mathbb{F}_{q}^{n}$ (excluding the all-zero vector). We say that two subspaces are disjoint if they have only trivial intersection. A $k$-spread in $\mathcal{G}_{q}(n, k)$ exists if and only if $k$ divides $n$ [37]. Clearly, a $k$-spread is a constant dimension code in $\mathcal{G}_{q}(n, k)$ with the maximal possible minimum distance $d_{S}=2 k$. A partition of all $k$-dimensional subspaces of $\mathcal{G}_{q}(n, k)$ into disjoint $k$-spreads is called a $k$-parallelism. The following construction is presented for $q=2$. 
Construction III: Let $\mathbb{T}$ be an $\operatorname{STD}_{2}(3,4,4)$ obtained from an $(8,4,2)_{2} \mathbb{C}^{\mathrm{MRD}}$. We will generate a new code $\mathbb{C}$ which contains $\mathbb{C}^{\mathrm{MRD}}$. The following new codewords (blocks) will form the elements of $\mathbb{C} \backslash \mathbb{C}^{\mathrm{MRD}}$.

Let $\mathcal{B}_{1}, \mathcal{B}_{2}, \ldots, \mathcal{B}_{7}$ be a partition of all the subspaces of $\mathcal{G}_{2}(4,2)$ into seven 2 -spreads, each one of size 5 , i.e., a well known 2-parallelism in $\mathcal{G}_{2}(4,2)$ [3], [5], [54]. For each $i$, $1 \leq i \leq 7$, and each two subspaces $Z, Z^{\prime} \in \mathcal{B}_{i}\left(Z^{\prime}\right.$ can be equal to $Z$ ) we write $Z=\left\{v_{0}=\mathbf{0}, v_{1}, v_{2}, v_{3}\right\}$ and $Z^{\prime}=\left\{v_{0}^{\prime}=\mathbf{0}, v_{1}^{\prime}, v_{2}^{\prime}, v_{3}^{\prime}\right\}$, where $v_{t}, v_{t}^{\prime} \in \mathbb{F}_{2}^{4}, 0 \leq t \leq 3$, and $\mathbf{0}=(0000)$. The 2-dimensional subspace $Z$ has four cosets $Z_{0}=Z, Z_{1}, Z_{2}, Z_{3}$ in $\mathbb{F}_{2}^{4}$. We construct the following four codewords in $\mathbb{C} \backslash \mathbb{C}^{\mathrm{MRD}}$. The codewords are defined by fifteen nonzero vectors which are the nonzero vectors of a 4 -dimensional subspace as can be verified.

(C.1) $\left\{(\mathbf{0} \| u): u \in Z_{0} \backslash\{\boldsymbol{0}\}\right\} \cup\left\{\left(v_{1}^{\prime} \| y\right): y \in Z_{0}\right\}$

$$
\cup\left\{\left(v_{2}^{\prime}|| y\right): y \in Z_{0}\right\} \cup\left\{\left(v_{3}^{\prime}|| y\right): y \in Z_{0}\right\},
$$

(C.2) $\left\{(\mathbf{0} \| u): u \in Z_{0} \backslash\{\mathbf{0}\}\right\} \cup\left\{\left(v_{1}^{\prime} \| y\right): y \in Z_{1}\right\}$

$$
\cup\left\{\left(v_{2}^{\prime}|| y\right): y \in Z_{2}\right\} \cup\left\{\left(v_{3}^{\prime}|| y\right): y \in Z_{3}\right\},
$$

(C.3) $\left\{(\mathbf{0} \| u): u \in Z_{0} \backslash\{\mathbf{0}\}\right\} \cup\left\{\left(v_{1}^{\prime} \| y\right): y \in Z_{2}\right\}$

$$
\cup\left\{\left(v_{2}^{\prime}|| y\right): y \in Z_{3}\right\} \cup\left\{\left(v_{3}^{\prime}|| y\right): y \in Z_{1}\right\},
$$

(C.4) $\left\{(\mathbf{0} \| u): u \in Z_{0} \backslash\{\mathbf{0}\}\right\} \cup\left\{\left(v_{1}^{\prime} \| y\right): y \in Z_{3}\right\}$

$$
\cup\left\{\left(v_{2}^{\prime}|| y\right): y \in Z_{1}\right\} \cup\left\{\left(v_{3}^{\prime}|| y\right): y \in Z_{2}\right\} .
$$

In $\mathcal{G}_{2}(4,2)$ there are $\left[\begin{array}{l}4 \\ 2\end{array}\right]_{2}=35$ 2-dimensional subspaces, and hence there are 35 different choices for $Z$. Since the size of a spread is 5 , it follows that there are 5 different choices for $Z^{\prime}$. Thus, there are a total of $35 \cdot 5 \cdot 4=700$ codewords in $\mathbb{C} \backslash \mathbb{C}^{\mathrm{MRD}}$ generated in this way. In addition to these 700 codewords we add a codeword which contains all the points of $\mathbb{V}_{0}^{(n, k)}$.

Example 6: A partition of $\mathcal{G}_{2}(4,2)$ into seven spreads is given in the following table, where each row corresponds to a spread.

TABLE IV

PARTITION OF $\mathcal{G}_{2}(4,2)$

\begin{tabular}{|l|l|l|l|l|l|}
\hline $\mathcal{B}_{1}$ & 1000 & 1010 & 1011 & 1001 & 0010 \\
& 1100 & 0101 & 0110 & 0111 & 0001 \\
& 1111 & 1101 & 1110 & 0011 \\
\hline \multirow{3}{*}{$\mathcal{B}_{2}$} & 0000 & 0100 & 1011 & 1001 & 1101 \\
& 1010 & 0001 & 0111 & 0110 & 0011 \\
\hline \multirow{3}{*}{$\mathcal{B}_{3}$} & 1000 & 1001 & 1100 & 1111 & 1110 \\
\hline & 0110 & 0100 & 0011 & 0101 & 1010 \\
& 1110 & 1101 & 1111 & 0111 & 0001 \\
$\mathcal{B}_{4}$ & 1000 & 1011 & 1100 & 1010 & 0101 \\
& 0001 & 0100 & 0010 & 0111 & 0011 \\
& 1001 & 1111 & 1110 & 1101 & 0110 \\
\hline \multirow{3}{*}{$\mathcal{B}_{5}$} & 1000 & 0100 & 1010 & 1001 & 1110 \\
& 0101 & 0011 & 0110 & 0010 & 0001 \\
& 1101 & 0111 & 1100 & 1011 & 1111 \\
\hline \multirow{2}{*}{$\mathcal{B}_{6}$} & 1000 & 0100 & 1100 & 1000 & 1011 \\
& 0111 & 0010 & 0001 & 0011 & 0101 \\
& 1111 & 0110 & 1101 & 1001 & 1110 \\
\hline \multirow{2}{*}{$\mathcal{B}_{7}$} & 1000 & 1010 & 1001 & 1101 & 0110 \\
& 0011 & 0100 & 0101 & 0010 & 0001 \\
& 1011 & 1110 & 1100 & 1111 & 0111 \\
\hline
\end{tabular}

We illustrate the idea of Construction III by considering one 2 -spread and a coset of one element of the spread. Let $\mathcal{B}_{1}=$ $\left\{Z^{0}, Z^{1}, Z^{2}, Z^{3}, Z^{4}\right\}$ be a spread given by the first row of the table, i.e., $Z^{0}=\langle(1000),(0100)\rangle, Z^{1}=\langle(1010),(0101)\rangle$, $Z^{2}=\langle(1011),(0110)\rangle, Z^{3}=\langle(1001),(0111)\rangle, Z^{4}=$ $\langle(0010),(0001)\rangle$. The four cosets of $Z^{0}$ are given by

$$
\begin{gathered}
Z_{0}=Z^{0}=\{(0000),(1000),(0100),(1100)\}, \\
Z_{1}=\{(0001),(1001),(0101),(1101)\}, \\
Z_{2}=\{(0010),(1010),(0110),(1110)\}, \\
Z_{3}=\{(0011),(1011),(0111),(1111)\} .
\end{gathered}
$$

For the pair $Z^{0}, Z^{1}$, the following four subspaces $C_{1}$, $C_{2}, C_{3}$, and $C_{4}$, belong to the code and correspond to the four types of the codewords, where $C_{i}$ corresponds to (C. $i$ ), $1 \leq i \leq 4$, and for every coset of $Z^{0}$ we use a different color.

\begin{tabular}{|c|c|c|c|}
\hline$C_{1}$ & $C_{2}$ & $C_{3}$ & $C_{4}$ \\
\hline 00001000 & 00001000 & 00001000 & 00001000 \\
00000100 & 00000100 & 00000100 & 00000100 \\
00001100 & 00001100 & 00001100 & 00001100 \\
10100000 & 10100001 & 10100010 & 10100011 \\
10101000 & 10101001 & 10101010 & 10101011 \\
10100100 & 10100101 & 10100110 & 10100111 \\
10101100 & 10101101 & 10101110 & 10101111 \\
01010000 & 01010010 & 01010011 & 01010001 \\
01011000 & 01011010 & 01011011 & 01011001 \\
01010100 & 01010110 & 01010111 & 01010101 \\
01011100 & 01011110 & 01011111 & 01011101 \\
11110000 & 11110011 & 11110001 & 11110010 \\
11111000 & 11111011 & 11111001 & 11111010 \\
11110100 & 11110111 & 11110101 & 11110110 \\
11110100 & 11111111 & 11111101 & 11111110 \\
\hline
\end{tabular}

Theorem 18: Construction III generates an $\left(8,2^{12}+\right.$ $701,4,4)_{2}$ constant dimension code $\mathbb{C}$ which attains the bound of Theorem 11 and contains an $(8,4,2)_{2} \mathbb{C}^{\mathrm{MRD}}$.

Proof: First, we observe that the four types of codewords given in the construction are indeed 4-dimensional subspaces of $\mathbb{F}_{2}^{8}$. Each one of the codewords contains 15 different onedimensional subspaces, and hence each codeword contains 15 different nonzero vectors of $\mathbb{F}_{2}^{8}$. It is easy to verify that all these vectors are closed under addition in $\mathbb{F}_{2}$, thus each constructed codeword is a 4-dimensional subspace of $\mathbb{F}_{2}^{8}$.

To prove that for each two codewords $X, Y \in \mathbb{C}$, we have $d_{S}(X, Y) \geq 4$, we distinguish between three cases:

- Case 1: $X, Y \in \mathbb{C}^{\mathrm{MRD}}$. Since the minimum distance of $\mathbb{C}^{\mathrm{MRD}}$ is 4 , we have that $d_{S}(X, Y) \geq 4$.

- Case 2: $X \in \mathbb{C}^{\mathrm{MRD}}$ and $Y \in \mathbb{C} \backslash \mathbb{C}^{\mathrm{MRD}}$. The codewords of $\mathbb{C}^{\mathrm{MRD}}$ forms the blocks of an $\operatorname{STD}_{2}(3,4,4), \mathbb{T}$, and hence meet each group in exactly one point. Each codeword of $\mathbb{C} \backslash \mathbb{C}^{\mathrm{MRD}}$ meets exactly three groups of $\mathbb{T}$. Hence, $\operatorname{dim}(X \cap Y) \leq 2$ for each $X \in \mathbb{C}^{\mathrm{MRD}}$ and $Y \in \mathbb{C} \backslash \mathbb{C}^{\mathrm{MRD}}$, therefore, $d_{S}(X, Y) \geq 4$. 
- Case 3: $X, Y \in \mathbb{C} \backslash \mathbb{C}^{\mathrm{MRD}}$. If $X$ and $Y$ have exactly three points in common in $\mathbb{V}_{0}^{(8,4)}$ (which correspond to a 2dimensional subspace contained in $\mathcal{L}_{0}^{(8,4)}$ ), then they are disjoint in all the groups of $\mathbb{T}$. This is due to the fact that the points of $X$ in $\mathbb{V}^{8}$ and the point of $Y$ in $\mathbb{V}^{8}$ correspond to either different cosets, or different blocks in the same spread. If $X$ and $Y$ have exactly one point in common in $\mathbb{V}_{0}^{(8,4)}$, then they have at most two points in common in at most one group of $\mathbb{T}$. Thus, $d_{S}(X, Y) \geq 4$. $(8,4,2)_{2} \mathbb{C}^{\mathrm{MRD}}$ contains $2^{12}$ codewords. As explained in the construction, there are 701 codewords in $\mathbb{C} \backslash \mathbb{C}^{\mathrm{MRD}}$. Thus, in the constructed code $\mathbb{C}$ there are $2^{12}+701=4797$ codewords.

Thus, the code attains the bound of Theorem 11.

Remark 5: Theorem 18 implies that $A_{2}(8,4,4) \geq 4797$ (the previous best known lower bound was $A_{2}(8,4,4) \geq$ 4605 [39]).

Remark 6: Construction III can be easily generalized for all prime powers $q \geq 2$, since there is a 2-parallelism in $\mathcal{G}_{q}(n, 2)$ for all such $q$, where $n$ is power of 2 [5]. Thus, from this construction we can obtain a $(8, M, 4,4)_{q}$ code with $M=$ $q^{12}+\left[\begin{array}{l}4 \\ 2\end{array}\right]_{q}\left(q^{2}+1\right) q^{2}+1$, since the size of a 2 -spread in $\mathcal{G}_{q}(4,2)$ is $q^{2}+1$ and there are $q^{2}$ different cosets of a 2 dimensional subspace in $\mathbb{F}_{q}^{4}$.

In the following table we compare the size of codes obtained by Construction III and its generalizations for large $q$ (denoted by $\mathbb{C}_{n e w}$ ) with the size of the largest previously known codes (denoted by $\mathbb{C}_{o l d}$ ) and with the upper bound (2) (for $n=8$ and $k=4$ ).

\begin{tabular}{|c|c|c|c|}
\hline$q$ & $\left|\mathbb{C}_{\text {old }}\right|$ & $\left|\mathbb{C}_{\text {new }}\right|$ & upper bound 2] \\
\hline \hline 2 & $2^{12}+509[39]$ & $2^{12}+701$ & $2^{12}+2381$ \\
\hline 3 & $3^{12}+8137[13]$ & $3^{12}+11701$ & $3^{12}+95941$ \\
\hline 4 & $4^{12}+72529[13]$ & $4^{12}+97105$ & $4^{12}+1467985$ \\
\hline
\end{tabular}

Remark 7: In general, the existence of $k$-parallelism in $\mathcal{G}_{q}(n, k)$ is an open problem. It is known that 2-parallelism exists for $q=2$ and all $n$ [3], [54], and for each prime power $q$, where $n$ is power of 2 [5]. There is also a 3-parallelism for $q=2$ and $n=6$ [36]. Thus we believe that Construction III can be generalized to a larger family of parameters assuming that there exists a corresponding parallelism.

\section{LINEAR CODES DERIVED FROM LIFTED MRD CODES}

A lifted MRD code and the transversal design derived from it can also be used to construct a linear code in the Hamming space. In this section we study the properties of such a linear code, whose parity-check matrix is an incidence matrix of a transversal design derived from a lifted MRD code. Some of the results presented in this section generalize the results given in [24]. In particular, the lower bounds on the minimum distance and the bounds on the dimension of codes derived from lifted MRD codes with $k-\delta=1$ coincide with the bounds on LDPC codes from partial geometries considered in [24].

For each codeword $X$ of an $(n, k, \delta)_{q} \mathbb{C}^{\mathrm{MRD}}$ we define its binary incidence vector $x$ of length $\left|\mathbb{V}^{n}\right|=\frac{q^{n}-q^{n-k}}{q-1}$ as follows: $x_{A}=1$ if and only if the point (one-dimensional subspace) $A \in \mathbb{V}^{n}$ is contained in $X$.

Let $H$ be the $\left|\mathbb{C}^{\mathrm{MRD}}\right| \times\left|\mathbb{V}^{n}\right|$ binary matrix whose rows are the incidence vectors of the codewords of $\mathbb{C}^{\mathrm{MRD}}$. By Theorem 6, this matrix $H$ is the incidence matrix of a $\operatorname{TD}_{\lambda}\left(\frac{q^{k}-1}{q-1}, q^{n-k}\right)$, with $\lambda=q^{(n-k)(k-\delta-1)}$. Note that the rows of the incidence matrix $H$ correspond to the blocks of the transversal design, and the columns of $H$ correspond to the points of the transversal design. If $\lambda=1$ in such a design (or, equivalently, $\delta=k-1$ for $\mathbb{C}^{\mathrm{MRD}}$ ), then $H^{T}$ is an incidence matrix of a net, the dual structure to the transversal design [31, p. 243].

An $[N, K, d]$ linear code is a linear subspace of dimension $K$ of $\mathbb{F}_{2}^{N}$ with minimum Hamming distance $d$. Let $C$ be the linear code with the parity-check matrix $H$, and let $C^{T}$ be the linear code with the parity-check matrix $H^{T}$.

The code $C$ has length $\frac{q^{n}-q^{n-k}}{q-1}$ and the code $C^{T}$ has length $q^{(n-k)(k-\delta+1)}$. By Corollary 3 each column of $H$ has $q^{(n-k)(k-\delta)}$ ones; since each $k$-dimensional subspace contains $\frac{q^{k}-1}{q-1}$ one-dimensional subspaces, each row has $\frac{q^{k}-1}{q-1}$ ones.

Remark 8: Note that if $\delta=k$, then the column weight of $H$ is one. Hence, the minimum distance of $C$ is 2. Moreover, $C^{T}$ consists only of the all-zero codeword. Thus, these codes are not interesting and hence in the sequel we assume that $\delta \leq k-1$.

Lemma 19: The matrix $H$ obtained from an $(n, k, \delta)_{q}$ $\mathbb{C}^{\mathrm{MRD}}$ code can be decomposed into blocks, where each block is a $q^{n-k} \times q^{n-k}$ permutation matrix.

Proof: It follows from Lemma 5 that the related transversal design is resolvable. In each parallel class each element of $\mathbb{V}^{n}$ is contained in exactly one codeword of $\mathbb{C}^{\mathrm{MRD}}$. Each class has $q^{n-k}$ codewords, each group has $q^{n-k}$ points, and each codeword meets each group in exactly one point. This implies that the $q^{n-k}$ rows of $H$ related to each such class can be decomposed into $\frac{q^{k}-1}{q-1} q^{n-k} \times q^{n-k}$ permutation matrices.

Example 7: A $[12,4,6]$ code $C$ and a $[16,8,4]$ code $C^{T}$ are obtained from the $(4,16,2,2)_{2}$ lifted MRD code $\mathbb{C}^{\mathrm{MRD}}$. The incidence matrix for corresponding transversal design $\mathrm{TD}_{1}(3,4)$ (see Example 2) is given by the following $16 \times 12$ matrix. The four rows above this matrix represent the column vectors for the points of the design.

\begin{tabular}{|c|c|c|}
\hline $\begin{array}{llll}0 & 0 & 0 & 0\end{array}$ & $\begin{array}{llll}1 & 1 & 1 & 1\end{array}$ & $\begin{array}{llll}1 & 1 & 1 & 1\end{array}$ \\
\hline $\begin{array}{llll}1 & 1 & 1 & 1\end{array}$ & $\begin{array}{llll}0 & 0 & 0 & 0\end{array}$ & $\begin{array}{llll}1 & 1 & 1 & 1\end{array}$ \\
\hline $\begin{array}{lllll}0 & 0 & 1 & 1\end{array}$ & $\begin{array}{lllll}0 & 0 & 1 & 1\end{array}$ & $\begin{array}{lllll}0 & 0 & 1 & 1\end{array}$ \\
\hline $\begin{array}{lllll}0 & 1 & 0 & 1\end{array}$ & $\begin{array}{llll}0 & 1 & 0 & 1\end{array}$ & $\begin{array}{llll}0 & 1 & 0 & 1\end{array}$ \\
\hline 10000 & 1000 & 1000 \\
\hline $\begin{array}{llll}0 & 1 & 0 & 0\end{array}$ & $\begin{array}{llll}0 & 0 & 0 & 1\end{array}$ & $\begin{array}{lllllll}0 & 0 & 1 & 0\end{array}$ \\
\hline $\begin{array}{lllll}0 & 0 & 1 & 0\end{array}$ & $\begin{array}{llll}0 & 1 & 0 & 0\end{array}$ & $\begin{array}{lllll}0 & 0 & 0 & 1\end{array}$ \\
\hline $\begin{array}{lllll}0 & 0 & 0 & 1\end{array}$ & $\begin{array}{lllllll}0 & 0 & 1 & 0\end{array}$ & $\begin{array}{llll}0 & 1 & 0 & 0\end{array}$ \\
\hline 10000 & $\begin{array}{lllll} & 1 & 0 & 0\end{array}$ & $\begin{array}{lllll} & 1 & 0 & 0\end{array}$ \\
\hline $\begin{array}{llll}0 & 1 & 0 & 0\end{array}$ & $\begin{array}{llll}0 & 0 & 1 & 0\end{array}$ & $\begin{array}{lllll}0 & 0 & 0 & 1\end{array}$ \\
\hline $\begin{array}{lllll}0 & 0 & 1 & 0\end{array}$ & 1000 & $\begin{array}{llll}0 & 0 & 1 & 0\end{array}$ \\
\hline $\begin{array}{llll}0 & 0 & 0 & 1\end{array}$ & $\begin{array}{llll}0 & 0 & 0 & 1\end{array}$ & 1000 \\
\hline 100000 & $\begin{array}{lllll}0 & 0 & 1 & 0\end{array}$ & $\begin{array}{lllll}0 & 0 & 1 & 0\end{array}$ \\
\hline $\begin{array}{llll}0 & 1 & 0 & 0\end{array}$ & $\begin{array}{llll}0 & 1 & 0 & 0\end{array}$ & 1000 \\
\hline $\begin{array}{lllll}0 & 0 & 1 & 0\end{array}$ & $\begin{array}{lllll}0 & 0 & 0 & 1\end{array}$ & $\begin{array}{llll}0 & 1 & 0 & 0\end{array}$ \\
\hline $\begin{array}{lllll}0 & 0 & 0 & 1\end{array}$ & 1000 & $\begin{array}{llll}0 & 0 & 0 & 1\end{array}$ \\
\hline 100000 & $\begin{array}{llll}0 & 0 & 0 & 1\end{array}$ & $\begin{array}{lllll}0 & 0 & 0 & 1\end{array}$ \\
\hline $\begin{array}{llll}0 & 1 & 0 & 0\end{array}$ & 1000 & 0100 \\
\hline $\begin{array}{lllll}0 & 0 & 1 & 0\end{array}$ & $\begin{array}{llll}0 & 0 & 1 & 0\end{array}$ & 1000 \\
\hline $\begin{array}{llll}0 & 0 & 0 & 1\end{array}$ & $\begin{array}{llll}0 & 1 & 0 & 0\end{array}$ & $\begin{array}{lllll}0 & 0 & 1 & 0\end{array}$ \\
\hline
\end{tabular}


Corollary 7: All the codewords of the code $C$, associated with the parity-check matrix $H$, and of the code $C^{T}$, associated with the parity-check matrix $H^{T}$, have even weights.

Corollary 8: The minimum Hamming distance $d$ of $C$ and the minimum Hamming distance $d^{T}$ of $C^{T}$ are upper bounded by $2 q^{n-k}$.

To obtain a lower bound on the minimum Hamming distance of these codes we need the following theorem known as the Tanner bound [45].

Theorem 20: The minimum distance, $d_{\min }$, of a linear code defined by an $m \times n$ parity-check matrix $\mathcal{H}$ with constant row weight $\rho$ and constant column weight $\gamma$ satisfy

T1: $\quad d_{\min } \geq \frac{n\left(2 \gamma-\mu_{2}\right)}{\gamma \rho-\mu_{2}}$,

T2: $\quad d_{\min } \geq \frac{2 n\left(2 \gamma+\rho-2-\mu_{2}\right)}{\rho\left(\gamma \rho-\mu_{2}\right)}$,

where $\mu_{2}$ is the second largest eigenvalue of $\mathcal{H}^{T} \mathcal{H}$.

To obtain a lower bound on $d$ and $d^{T}$ we need to find the second largest eigenvalue of $H^{T} H$ and $H H^{T}$, respectively. Note that since the set of eigenvalues of $H^{T} H$ and the set of eigenvalues of $H H^{T}$ are the same, it is sufficient to find only the eigenvalues of $H^{T} H$.

The following lemma is derived from [9, p. 563].

Lemma 21: Let $\mathcal{H}$ be an incidence matrix for $\operatorname{TD}_{\lambda}(k, m)$. The eigenvalues of $\mathcal{H}^{T} \mathcal{H}$ are $r k, r$, and $r k-k m \lambda$ with multiplicities $1, k(m-1)$, and $k-1$, respectively, where $r$ is a number of blocks that are incident with a given point.

By Corollary $3, r=q^{(n-k)(k-\delta)}$ in $\operatorname{TD}_{\lambda}\left(\frac{q^{k}-1}{q-1}, q^{n-k}\right)$ with $\lambda=q^{(n-k)(k-\delta-1)}$. Thus, from Lemma 21 we obtain the spectrum of $H^{T} H$.

Corollary 9: The eigenvalues of $H^{T} H$ are $q^{(n-k)(k-\delta)} \frac{q^{k}-1}{q-1}, q^{(n-k)(k-\delta)}$, and 0 with multiplicities 1 , $\frac{q^{k}-1}{q-1}\left(q^{n-k}-1\right)$, and $\frac{q^{k}-1}{q-1}-1$, respectively.

Now, by Theorem 20 and Corollary 9, we have Corollary 10:

$$
\begin{gathered}
d \geq \frac{q^{n-k}\left(q^{k}-1\right)}{q^{k}-q}, \\
d^{T} \geq\left\{\begin{array}{cc}
2^{k} & \delta=k-1, q=2, k=n-k \\
4 q^{(n-k)(\delta-k+1)} & \text { otherwise }
\end{array}\right.
\end{gathered}
$$

Proof: By Corollary 9, the second largest eigenvalue of $H^{T} H$ is $\mu_{2}=q^{(n-k)(k-\delta)}$. We apply Theorem 20(T1) to obtain

$d \geq \frac{q^{n-k} \frac{q^{k}-1}{q-1}\left(2 q^{(n-k)(k-\delta)}-q^{(n-k)(k-\delta)}\right)}{q^{(n-k)(k-\delta)} \frac{q^{k}-1}{q-1}-q^{(n-k)(k-\delta)}}=\frac{q^{n-k}\left(q^{k}-1\right)}{q^{k}-q}$.

By using Theorem 20 we also obtain lower bounds on $d^{T}$ :

$$
\begin{gathered}
d^{T} \geq \frac{q^{n-k}\left(2 \frac{q^{k}-1}{q-1}-q^{(n-k)(k-\delta)}\right)}{\frac{q^{k}-1}{q-1}-1}, \\
d^{T} \geq 4 q^{(n-k)(\delta-k+1)} .
\end{gathered}
$$

Note that the expression in (4) is negative for $\delta<k-1$. For $\delta=k-1$ with $k=n-k$ and $q=2$, the bound in (4) is larger than the bound in (5). Thus, we have $d^{T} \geq 2^{k}$, if $\delta=k-1, q=2$, and $k=n-k$; and $d^{T} \geq 4 q^{(n-k)(\delta-k+1)}$, otherwise.

We use the following result derived from [25, Theorem 1] to improve the lower bound on $d^{T}$.

Lemma 22: Let $\mathcal{H}$ be an incidence matrix of blocks (rows) and points (columns) such that each block contains exactly $\kappa$ points, and each pair of distinct blocks intersects in at most $\gamma$ points. If $d_{\mathcal{H}^{T}}$ is a minimum distance of a code with the parity-check matrix $\mathcal{H}^{T}$ then

$$
d_{\mathcal{H}^{T}} \geq \frac{\kappa}{\gamma}+1
$$

Corollary 11: $d^{T} \geq \frac{q^{k}-1}{q^{k-\delta}-1}+1$.

Proof: By Lemma 22, with $\kappa=\frac{q^{k}-1}{q-1}$ and $\gamma=\frac{q^{k-\delta}-1}{q-1}$, since any two codewords in a lifted MRD code intersect in at most $(k-\delta)$-dimensional subspace, we have the following lower bound on the minimum distance of $C^{T}$

$$
d^{T} \geq \frac{\left(q^{k}-1\right) /(q-1)}{\left(q^{k-\delta}-1\right) /(q-1)}+1=\frac{q^{k}-1}{q^{k-\delta}-1}+1 .
$$

Obviously, for all $\delta \leq k-1$, this bound is larger or equal than the bound of Corollary 10, and thus the result follows.

Let $\operatorname{dim}(C)$ and $\operatorname{dim}\left(C^{T}\right)$ be the dimensions of $C$ and $C^{T}$, respectively. To obtain the lower and upper bounds on $\operatorname{dim}(C)$ and $\operatorname{dim}\left(C^{T}\right)$ we need the following basic results from linear algebra [22]. For a matrix $A$ over a field $\mathbb{F}$, let $\operatorname{rank}_{\mathbb{F}}(A)$ denotes the rank of $A$ over $\mathbb{F}$.

Lemma 23: Let $A$ be a $\rho \times \eta$ matrix, and let $\mathbb{R}$ be the field of real numbers. Then

- $\operatorname{rank}_{\mathbb{R}}(A)=\operatorname{rank}_{\mathbb{R}}\left(A^{T}\right)=\operatorname{rank}_{\mathbb{R}}\left(A^{T} A\right)$.

- If $\rho=\eta$ and $A$ is a symmetric matrix with the eigenvalue 0 of multiplicity $t$, then $\operatorname{rank}_{\mathbb{R}}(A)=\eta-t$.

Theorem 24:

$$
\begin{gathered}
\operatorname{dim}(C) \geq \frac{q^{k}-1}{q-1}-1, \\
\operatorname{dim}\left(C^{T}\right) \geq q^{(n-k)(k-\delta+1)}-\frac{q^{k}-1}{q-1}\left(q^{n-k}-1\right)-1 .
\end{gathered}
$$

Proof: First, we observe that $\operatorname{dim}(C)=\frac{q^{k}-1}{q-1} q^{n-k}-$ $\operatorname{rank}_{\mathbb{F}_{2}}(H)$, and $\operatorname{dim}\left(C^{T}\right)=q^{(n-k)(k-\delta+1)}-\operatorname{rank}_{\mathbb{F}_{2}}\left(H^{T}\right)$. Now we obtain an upper bound on $\operatorname{rank}_{\mathbb{F}_{2}}(H)=\operatorname{rank}_{\mathbb{F}_{2}}\left(H^{T}\right)$. Clearly, $\operatorname{rank}_{\mathbb{F}_{2}}(H) \leq \operatorname{rank}_{\mathbb{R}}(H)$. By Corollary 9 , the multiplicity of an eigenvalue 0 of $H^{T} H$ is $\frac{q^{k}-1}{q-1}-1$. Hence by Lemma 23] $\operatorname{rank}_{\mathbb{F}_{2}}(H) \leq \operatorname{rank}_{\mathbb{R}}(H)=\operatorname{rank}_{\mathbb{R}}\left(H^{T} H\right)=$ $\frac{q^{k}-1}{q-1} q^{n-k}-\left(\frac{q^{k}-1}{q-1}-1\right)$. Thus, $\operatorname{dim}(C) \geq \frac{q^{k}-1}{q-1} q^{n-k}-$ $\left(\frac{q^{k}-1}{q-1} q^{n-k}-\left(\frac{q^{k}-1}{q-1}-1\right)\right)=\frac{q^{k}-1}{q-1}-1$, and $\operatorname{dim}\left(C^{T}\right) \geq$ $q^{(n-k)(k-\delta+1)}-\frac{q^{k}-1}{q-1} q^{n-k}+\frac{q^{k}-1}{q-1}-1$.

Now, we obtain an upper bound on the dimension of the codes $C$ and $C^{T}$ for odd $q$.

Theorem 25: Let $q$ be a power of an odd prime number.

- If $\frac{q^{k}-1}{q-1}$ is odd, then $\operatorname{dim}(C) \leq \frac{q^{k}-1}{q-1}-1$ and $\operatorname{dim}\left(C^{T}\right) \leq$ $q^{(n-k)(k-\delta+1)}-\frac{q^{k}-1}{q-1}\left(q^{n-k}-1\right)-1$.

- If $\frac{q^{k}-1}{q-1}$ is even, then $\operatorname{dim}(C) \leq \frac{q^{k}-1}{q-1}$, and $\operatorname{dim}\left(C^{T}\right) \leq$ $q^{(n-k)(k-\delta+1)}-\frac{q^{k}-1}{q-1}\left(q^{n-k}-1\right)$. 
Proof: We compute the lower bound on $\operatorname{rank}_{\mathbb{F}_{2}}(H)$ to obtain the upper bound on the dimension of the codes $C$ and $C^{T}$. First, we observe that $\operatorname{rank}_{\mathbb{F}_{2}}(H) \geq \operatorname{rank}_{\mathbb{F}_{2}}\left(H^{T} H\right)$. By [8], the rank over $\mathbb{F}_{2}$ of an integral diagonalizable square matrix $A$ is lower bounded by the sum of the multiplicities of the eigenvalues of $A$ that do not vanish modulo 2 . We consider now $\operatorname{rank}_{\mathbb{F}_{2}}\left(H^{T} H\right)$. By Corollary 9 the second eigenvalue of $H^{T} H$ is always odd for odd $q$. If $\frac{q^{k}-1}{q-1}$ is odd, then the first eigenvalue of $H^{T} H$ is also odd. Hence, we sum the multiplicities of the first two eigenvalues to obtain $\operatorname{rank}_{\mathbb{F}_{2}}\left(H^{T} H\right) \geq 1+\frac{q^{k}-1}{q-1}\left(q^{n-k}-1\right)$. If $\frac{q^{k}-1}{q-1}$ is even, then the first eigenvalue is even, and hence we take only the multiplicity of the second eigenvalue to obtain $\operatorname{rank}_{\mathbb{F}_{2}}\left(H^{T} H\right) \geq \frac{q^{k}-1}{q-1}\left(q^{n-k}-1\right)$. The result follows now from the fact that the dimension of a code is equal to the difference between its length and $\operatorname{rank}_{\mathbb{F}_{2}}(H)$.

Remark 9: For even values of $q$ the method used in the proof for Theorem 25 leads to a trivial result, since in this case all the eigenvalues of $H^{T} H$ are even and thus by [8] we have $\operatorname{rank}_{\mathbb{F}_{2}}\left(H^{T} H\right) \geq 0$. But clearly, by Lemma 19 we have $\operatorname{rank}_{\mathbb{F}_{2}}(H) \geq q^{n-k}$. Thus, for even $q, \operatorname{dim}(C) \leq \frac{q^{k}-1}{q-1} q^{n-k}-$ $q^{n-k}=q^{n-k}\left(\frac{q^{k}-1}{q-1}-1\right)$, and $\operatorname{dim}\left(C^{T}\right)=q^{(n-k)(k-\delta+1)}-$ $q^{n-k}$.

Note that for odd $q$ and odd $\frac{q^{k}-1}{q-1}$ the lower and the upper bounds on the dimension of $C$ and $C^{T}$ are the same. Therefore, we have the following corollary.

Corollary 12: For odd $q$ and odd $\frac{q^{k}-1}{q-1}$ the dimensions $\operatorname{dim}(C)$ and $\operatorname{dim}\left(C^{T}\right)$ of the codes $C$ and $C^{T}$, respectively, satisfy $\operatorname{dim}(C)=\frac{q^{k}-1}{q-1}-1$, and $\operatorname{dim}\left(C^{T}\right)=q^{(n-k)(k-\delta+1)}-$ $\frac{q^{k}-1}{q-1} q^{n-k}+\frac{q^{k}-1}{q-1}-1$.

Finally, $C$ and $C^{T}$ can be also viewed as LDPC codes obtained from designs [2], [23], [24], [25], [28], [29], [30], [46], [50], [51], [55]. Some preliminary results in this direction can be found in [38], [40].

\section{CONCLUSIONS AND FUtURE RESEARCH}

Lifted MRD codes are considered. Properties of these codes, especially when viewed as transversal designs are proved. Based on this design new upper bounds and constructions for constant dimension codes which contain lifted MRD codes as subcodes are given. The incidence matrix of the design (which represents also the codewords of the lifted MRD code) is considered as a parity-check matrix of a linear code in the Hamming space. Properties of these linear codes are proved. We conclude with a list of open problems for future research.

1) What are the general upper bounds on a size of an $(n, M, 2 \delta, k)_{q}$ code which contains a lifted MRD code?

2) Are the upper bounds of Theorems 10 and 11 and related bounds for other parameters attained for all parameters?

3) Can the codes constructed in Constructions I, II, and III be used, in a recursive method, to obtain new bounds on $\mathcal{A}_{q}(n, d, k)$ for larger $n$ ?

4) One of the main research problems is to improve the lower bounds on $\mathcal{A}_{q}(n, d, k)$, with codes which do not contain the lifted MRD codes. Only such codes can close the gap between the lower and the upper bounds on $\mathcal{A}_{q}(n, d, k)$ for small $q$ and small $d$ (e.g. the seven codes for $k=3$ mentioned in the Introduction).

5) Which properties have LDPC codes obtained from lifted MRD codes? The bounds given in Section VI is only a first step in this direction. In addition, we would like to know the performance of these codes with various decoding algorithms [11], [34].

\section{REFERENCES}

[1] R. Ahlswede, H. K. Aydinian, and L. H. Khachatrian, "On perfect codes and related concepts," Designs, Codes, Crypt., vol. 22, pp. 221-237, 2001 .

[2] B. Ammar, B. Honary, Y. Kou, J. Xu, and S. Lin, "Construction of lowdensity parity-check codes based on balanced incomplete block designs," IEEE Trans. Inform. Theory, vol. 50, no. 6, pp. 1257-1568, Jun. 2004.

[3] R.D. Baker, "Partitioning the planes of $A G_{2 m}(2)$ into 2-designs", Discrete MAth, vol. 15, pp. 205-211, 1976.

[4] E. R. Berlekamp, "The technology of error-correcting codes", Proc. IEEE, vol. 68, pp. 564-593, May 1980

[5] A. Beutelspacher, "On parallelisms in finite projective spaces", Geometriae Dedicata, vol. 3, pp. 35-45, 1974.

[6] S. Blackburn and T. Etzion, "The Asymptotic Behavior of Grassmannian Codes", IEEE Trans. Inform. Theory, to appear (see also arxiv.org/abs/1111.2713).

[7] M. Braun, A. Kerber, and R. Laue, "Systematic construction of $q$-analogs of $t-(v, k, \lambda)$-designs", Designs, codes and Cryptography, vol. 34, pp. $55-70,2005$.

[8] A. E. Brouwer and C. A. van Eijl, "On the $p$-rank of strongly regular graphs", Algebra and Combinatorics, vol. 1, pp. 329-346, 1992.

[9] C. J. Colbourn and J. H. Dinitz, Handbook of Combinatorial Designs, Chapman and Hall/CRC, 2007 (Second edition).

[10] P. Delsarte, "Bilinear forms over a finite field, with applications to coding theory," Journal of Combinatorial Theory, Series A, vol. 25, pp. 226241, 1978.

[11] C. Di, D. Proietti, I. E. Telatar, T. J. Richardson, and R. L. Urbanke, "Finite-length analysis of low-density parity-check codes on the binary erasure channel", IEEE Trans. Inform. Theory, vol. 48, no. 6, 1570-1579, 2002.

[12] S. Dougherty, "Nets and their codes," Designs, Codes, Crypt., vol. 3, pp. 315-331, 1993.

[13] T. Etzion and N. Silberstein, "Error-correcting codes in projective space via rank-metric codes and Ferrers diagrams", IEEE Trans. Inform. Theory, vol. 55, no.7, pp. 2909-2919, July 2009.

[14] T. Etzion and A. Vardy, "Error-correcting codes in projective space", in proceedings of International Symposium on Information Theory, pp. 871-875, July 2008.

[15] T. Etzion and A. Vardy, "Error-correcting codes in projective space", IEEE Trans. Inform. Theory, vol.57, no. 2, pp. 1165-1173, February 2011.

[16] T. Etzion and A. Vardy, "On $q$-Analogs for Steiner Systems and Covering Designs", Advances in Mathematics of Communications, vol. 5, no. 2, pp. 161-176, 2011.

[17] E. M. Gabidulin, "Theory of codes with maximum rank distance," Problems of Information Transmission, vol. 21, pp. 1-12, July 1985.

[18] E. M. Gabidulin, A. V. Paramonov, and O. V. Tretjakov, "Ideals over a non-commutative ring and their application in cryptology," LNCS, vol. 573, pp. 482-489, 1991.

[19] M. Gadouleau and Z. Yan, "Packing and covering properties of subspace codes for error control in random linear network coding," IEEE Trans. Inform. Theory, vol. 56, no. 5, pp. 2097-2108, May 2010.

[20] M. Gadouleau and Z. Yan, "Constant-rank codes and their connection to constant-dimension codes," IEEE Trans. Inform. Theory, vol. 56, no. 7, pp. 3207-3216, July 2010.

[21] A. S. Hedayat, N. J. A. Sloane, and J. Stufken, Orthogonal arrays. Theory and applications, Springer, 1999.

[22] R. A. Horn, C. R. Johnson, Matrix analysis, Cambridge university press, 1985.

[23] S. Johnson and S. R. Weller, "Regular low-density parity-check codes from combinatorial designs," Proc. 2001 IEEE Inform. Theory Workshop, Cairns, Australia, pp. 90-92, Sept. 2-7, 2001.

[24] S. J. Johnson and S. R. Weller, "Codes for iterative decoding from partial geometries," IEEE Trans. on comm., vol. 52, pp. 236-243, 2004. 
[25] N. Kashyap and A. Vardy, "Stopping sets in codes from designs", Available: http://www.mast.queensu.ca/ nkashyap/Papers/stopsets.pdf preprint, 2003.

[26] R. Koetter and F. R. Kschischang, "Coding for errors and erasures in random network coding," IEEE Trans. Inform. Theory, vol. 54, no. 8, pp. 3579-3591, August 2008.

[27] A. Kohnert and S. Kurz, "Construction of large constant-dimension codes with a prescribed minimum distance," Lecture Notes in Computer Science, vol. 5393, pp.31-42, December 2008.

[28] Y. Kou, S. Lin, amd M. P. C. Fossorier, "Low density parity check codes based on finite geometries: a rediscovery and new results", IEEE Trans. Inform. Theory, vol. 47, no.7, pp. 2711-2736, 2001.

[29] S. Laendner and O. Milenkovic, "LDPC codes based on Latin squares: cyclic structure, stopping set, and trapping set analysis," IEEE Trans. Commun., vol. 55, no. 2, pp. 303-312, Feb. 2007.

[30] L. Lan, Y. Y. Tai, S. Lin, B. Memari and B. Honary, "New construction of quasi-cyclic LDPC codes based on special classes of BIBDs for the AWGN and binary erasure channels," IEEE Trans. Commun., vol 56, no.1, pp.39-48, Jam. 2008.

[31] J. H. van Lint and R. M. Wilson, A course in Combinatorics, Cambridge University Press, 2001 (second edition).

[32] P. Lusina, E. Gabidulin, and M. Bossert,"Maximum Rank Distance codes as space-time codes," IEEE Trans. Inform. Theory, vol. 49, pp. 27572760, Oct. 2003.

[33] F. Manganiello, E. Gorla, and J. Rosenthal, "Spread codes and spread decoding in network coding", in proc. of ISIT 2008, pp. 881-885.

[34] T. Richardson, "Error floors of LDPC codes," Proc. of the 41st Annual Allerton Conf. Commun., Constrol and Comp., Monticello, IL, pp.14261435, October 2003.

[35] R. M. Roth, "Maximum-rank array codes and their application to crisscross error correction," IEEE Trans. Inform. Theory, vol. 37, pp. 328-336, March 1991.

[36] J. Sarmiento, "On point-cyclic resolutions of the $2-(63,7,15)$ design associated with PG(5,2)," Graphs and Comb., vol. 18, pp. 621-632, 2002.

[37] M. Schwartz and T. Etzion, "Codes and anticodes in the Grassman graph", J. Combin. Theory, Ser. A, vol. 97, pp. 27-42, 2002.

[38] N. Silberstein, "Coding Theory and Projective Spaces," PhD thesis, Computer Science Dept., TechnionIsrael Inst. Technol., Haifa, 2011.

[39] N. Silberstein and T. Etzion, "Large constant dimension codes and lexicodes," Advances in Mathematics of Communications, vol. 5, no. 2, pp. 177-189, 2011

[40] N. Silberstein and T. Etzion, "Codes and Designs Related to Lifted MRD Codes", proceedings of International Symposium on Information Theory, pp. 2288-2292, July 2011.

[41] N. Silberstein, A. S. Rawat, S. Vishwanath, "Error resilience in distributed storage via rank-metric codes", preprint available http://arxiv.org/pdf/1202.0800v1.pdf 2012.

[42] D. Silva, F. R. Kschischang, and R. Koetter, "A rank-metric approach to error control in random network coding," IEEE Trans. Inform. Theory, vol. 54, pp. 3951-3967, September 2008.

[43] V. Skachek, "Recursive code construction for random networks," IEEE Trans. Inform. Theory, vol. 56, no. 3, pp. 1378-1382, March 2010.

[44] V.Y.F. Tan, L. Balzano, S.C. Draper, "Rank minimization over finite fields " proceedings of International Symposium on Information Theory, pp. 1195- 1199 , July 2011.

[45] R. M. Tanner, "Minimum Distance Bounds by Graph Analysis", IEEE Trans. Inform. Theory, vol. 47, 808-821, 2001.

[46] H. Tang, J. Xu, S. Lin, K. A. S. Abdel-Ghaffar, "Codes on Finite Geometries", IEEE Trans. Inform. Theory, vol. 51, no. 2, 572-596, 2005.

[47] S. Thomas, "Designs over finite fields," Geometriae Dedicata, vol. 21, pp. 237-242, 1987.

[48] A.-L. Trautmann and J. Rosenthal, "New improvements on the echelonFerrers construction", in proc. of Int. Symp. on Math. Theory of Networks and Systems, pp. 405-408, July 2010.

[49] A.-L. Trautmann, F. Manganiello, and J. Rosenthal, "Orbit codes- a new concept in the area of network coding", in proc. of Inf. Theory Workshop (ITW), pp. 1-4, 2010 IEEE, Dublin, Ireland, Ausgust 2010.

[50] B. Vasic, E. M. Kurtas, and A. Kuznetsov, "LDPC code based on mutually orthogonal Latin rectangles and their applications in perpendicular magnetic recording," IEEE Trans. Magn., vol. 38, no. 5, pp. 2346-2348, Sep. 2002.

[51] B. Vasic and O. Milenkovic, "Combinatorial construction of low-density parity-check codes for iterative decoding," IEEE Trans. Inf. Theory, vol. 50, no. 6, pp. 1156-1176, June 2004.
[52] H. Wang, and C. Xing and R. Safavi-Naini, "Linear authentication codes: bounds and constructions", IEEE Trans. on Inform. Theory, vol. 49, no. 4 pp. 866-872, 2003.

[53] S.-T.Xia and F.-W. Fu, "Johnson type bounds on constant dimension codes”, Designs, Codes, Crypto., vol. 50 no. 2, pp. 163 - 172, February 2009.

[54] G. Zaicev, V. Zinoviev, and N. Semakov, "Interrelation of Preparata and Hamming codes and extensions of Hamming codes to new double errorcorrecting codes ," The 2nd Int. Symp. Inform. Theory, pp. 257-263, 1971.

[55] L. Zhang, Q. Huang, S. Lin and K. Abdel-Ghaffar, Quasi-cyclic LDPC codes: an algebraic construction, rank analysis, and codes on Latin squares, IEEE Trans. Commu., vol. 58, no. 11, pp. 3126-3139, November 2010 . 